\title{
A Study of Some Tests of Uniformity and Their Performances
}

\author{
Tripakshi Borthakur ${ }^{1}$ and Bipin Gogoi ${ }^{2}$ \\ Research Scholar, Department of Statistics, Dibrugarh University, Dibrugarh, Assam \\ Professor, Department of Statistics, Dibrugarh University, Dibrugarh, Assam
}

\begin{abstract}
The uniform distribution appears due to natural random events or to the application of methods for transforming samples from any other distribution to samples with uniformly distributed values in the interval $(0,1)$. Thus, in order to test whether a samplecomes from a given distribution, one can test whether its transformed sample is distributed according to the uniform distribution or not. Several test procedures are developed to test the goodness of fit for uniformity. In this paper, we want to study the performance of eleven different tests for uniformity by considering different sample sizes as well as different alternatives. The results so obtained are displayed in various tables and graphs. Finally, conclusions are made on the basis of the results.
\end{abstract}

Keywords: Goodness of fit, level, Monte Carlo technique, power and uniform distribution.

\section{Introduction}

Goodness-of-fit tests are frequently used to decide if an observed sample $X_{i}, i=1,2, \ldots, n$ can be considered as a set of independent realizations of a given cumulative distribution function(cdf) $\mathrm{F}_{0}(\mathrm{x})$. More precisely, they are used to test the hypothesis $\mathrm{H}_{0}: \mathrm{F}=\mathrm{F}_{0}$, where $\mathrm{F}$ is the true cdf of the observations.Let us suppose that $\mathrm{F}_{0}$ is a Uniform distribution in the interval $[0,1]$. For testing uniformity, a number of authors proposed different statistical tests. The wide variety of tests is caused by frequent application of the uniform model in applications. This is not least defined by the fact that such a simple model makes it possible to solve problems based on analytical methods only.Uniform distribution is often used to describe the measurement errors of some devices or systems, which is not least due to the lack of information. Naturally, its unjustified use can cause problems.

The hypothesis of uniformity of random variables can be subjected to different statistical tests of that can be divided into two subsets. These include general goodness of fit tests used for uniformity and special tests meant only for the hypothesis of uniformity of the sample $X_{1}, X_{2}, \ldots, X_{n}$. Several tests are available for testing uniformity. Generally, a simple testable hypothesis of uniformity of the sample $X_{1}, X_{2}, \ldots, X_{n}$ of independent observations of a random value of $\mathrm{X}$ has the form: $\mathrm{H}_{0}: \mathrm{F}(\mathrm{x})=\mathrm{x}, \mathrm{x} \in[0,1]$. Most of the tests for the hypothesis of uniformity on the interval $[0,1]$ are based on the ordered samples $\mathrm{X}_{(1)}<\mathrm{X}_{(2)}<\ldots<\mathrm{X}_{(\mathrm{n})}$.In this paper, a simulation study is carried out to estimate the power of eleven different tests aiming to assess the validity of uniformity assumption on the unit interval $[0,1]$ by taking various sample sizes against five different alternative distributions.In the proposed study, section 2 represents a general description of the uniformity tests and the types of alternatives considered here, section 3 presents the simulation approach considered in the study and also the power results.

\subsection{Test Procedure:}

\section{Goodness -of- fit tests for uniformity}

Let $F$ be a continuous cumulative distribution function. Let $X_{1}, X_{2}, \ldots, X_{n}$ be a random sample from $F$. We are interested in testing $\mathrm{H}_{0}$ :Fwhere $\mathrm{U}[0,1]$ denotes the Uniform distribution in the interval $[0,1]$. Let $\mathrm{X}_{(1)}<\mathrm{X}_{(2)}<\ldots<\mathrm{X}_{(\mathrm{n})}$ be the ordered samples and $\bar{X}=\frac{1}{n} \sum_{i=1}^{n} X_{i}$ is the sample mean.

Generally, the use of nonparametric goodness of fit tests for composite hypotheses with regard to different parametric models of probability distribution laws is seriously complicated due to the dependence of test statistic distribution on a number of factors. But, in case of nonparametric tests used for uniformity, such type of problem does not arise. Therefore, in many situations, a sample that is belonged to some parametric law comes down to test the hypothesis of uniformity on the interval $[0,1]$.

In our proposed study, the eleven well-known test statistics $\mathrm{K}-\mathrm{S}\left(D_{n}\right), \operatorname{AD}\left(A_{n}^{2}\right), \operatorname{CvM}\left(W_{n}^{2}\right), \operatorname{Watson}\left(U_{n}^{2}\right)$, Sukhatme $\left(Q_{2}\right)$, Probability product $\left(Q_{1}\right)$, $\operatorname{Kuiper}\left(V_{n}\right)$, $\operatorname{Gini}\left(G_{n}\right)$, ZhangA $\left(Z_{A}\right), \operatorname{ZhangC}\left(Z_{C}\right)$ and $S_{n, A}^{1}$ defined in terms of a Uniform distribution function, have been considered. These statistics are described below: 


\subsubsection{Tests based on Empirical Distribution Function (EDF):}

The test statistics based on empirical distribution function (EDF) measure the discrepancy between the EDF, $\mathrm{F}_{\mathrm{n}}(\mathrm{x})$, and the distribution function, $\mathrm{F}(\mathrm{x})$, and are based on the vertical differences between these two functions. In our proposed study, the test statistics based on EDF which are considered herein may be subclassified into two groups:

(a) Supremum test statistics:

The most well known EDF statistic is $\mathrm{D}_{\mathrm{n}}$, introduced by Kolmogorov (1933) is given by

$$
\mathrm{D}_{\mathrm{n}}=\operatorname{Sup}_{-\infty<x<\infty}\left|F_{n}(x)-F(x)\right|=\max \left(D_{n}^{+}, D_{n}^{-}\right)
$$

Where $\quad D_{n}^{+}=\max _{1 \leq i \leq n}\left\{\frac{i}{n}-U_{i}\right\}$ and $D_{n}^{-}=\max _{1 \leq i \leq n}\left\{U_{i}-\frac{i-1}{n}\right\}$.

$D_{n}^{+}$and $D_{n}^{-}$are calculated as the largest vertical difference between $\mathrm{F}_{\mathrm{n}}(\mathrm{x})$ and $\mathrm{F}(\mathrm{x})$ when $\mathrm{F}_{\mathrm{n}}(\mathrm{x})$ is greater or smaller than $\mathrm{F}(\mathrm{x})$, respectively.

Kuiper (1960) introduced a related test statistic $V_{n}$ to be applied to observations on a circle since the value of $V_{n}$ does not depend on the origin choice. This test statistic is defined as

$$
\mathrm{V}_{\mathrm{n}}=D_{n}^{+}+D_{n}^{-} \text {. }
$$

Kuiper gave its asymptotic distribution. Tails distribution for small samples and critical points can be found in Stephens (1965) for both tails.

(b) Quadratic test statistics:

This wide family of discrepancy measures is given by theCramer-von Mises family $Q_{n}=n \int_{0}^{1}\left\{F_{n}(x)-F(x)\right\}^{2} \psi(x) d F(x)$, where $\psi(x)$ is a suitable function giving weights to $\left\{F_{n}(x)-F(x)\right\}^{2}$. When $\psi(x)=1$, this statistic is the Cramer-von Mises statistic $W_{n}^{2}$ and when $\psi(x)$ $=[\{\mathrm{F}(\mathrm{x})\}\{1-\mathrm{F}(\mathrm{x})\}]^{-1}$, this statistic is the Anderson-Darling (1954) statistic $A_{n}^{2}$. The test statisticisdefined as follows:

$$
\begin{gathered}
W_{n}^{2}=\sum_{i=1}^{n}\left\{U_{(i)}-\frac{2 i-1}{2 n}\right\}^{2}+\frac{1}{12 n} \\
A_{n=-n-\frac{1}{n}}^{2} \sum_{i=1}^{n}(2 i-1)\left[\ln U_{(i)}+\ln \left\{1-U_{(n+1-i)}\right\}\right]
\end{gathered}
$$

Lewis (1961) demonstrated that for $\mathrm{n}>3$, the asymptotic distribution of $A_{n}^{2}$ is a good approximation to its distribution and he proportionated a table containing the q-valuesof this asymptotic distribution for the lower tail, where $\mathrm{q}=P\left(A_{n}^{2}<t\right)$.

Watson (1961) proposed the statistic $U_{n}^{2}$, which is a modification of $W_{n}^{2}$, which is used with observations on a circle. This statistic is defined as

$$
U_{n}^{2}=W_{n}^{2}-n(\bar{U}-.5)^{2}
$$

The simulated Critical values of these tests are given in Table 1.

\subsubsection{Other approaches:}

\section{(a) The Sukhatme test:}

E.S.Pearson (1938) suggested that for the case when the alternative distribution may be concentrated in either end of the interval that we reject for the small values of

$$
Q_{2}=\prod_{i=1}^{n} U_{i}^{\prime}, \text { where } U_{i}^{\prime}=\left\{\begin{array}{l}
2 U_{i}, \text { if } 0<U_{i} \leq 1 / 2 \\
2\left(1-U_{i}\right), \text { if } 1 / 2<U_{i}<1
\end{array}\right\}
$$

The test is easily carried out by rejecting for large values of

$$
-2 \ln Q_{2}=-2 \sum_{i=1}^{n} \ln U_{i}^{\prime}
$$


which also has a chi-square distribution with $2 \mathrm{n}$ degrees of freedom. This test is a Uniformly Most Powerful(UMP) test for the alternative class of densities

$$
\begin{aligned}
h(y, v) & =(1-v) y^{-v}, 0<y \leq 1 / 2 \\
& =(1-v)(1-y)-v, 1 / 2<y<1,0<v<1
\end{aligned}
$$

(b) Pearson's Probability Product test:

Karl Pearson probability product test $\mathrm{Q}_{1}$ was proposed by Pearson in 1933 which is defined as

$$
\mathrm{Q}_{1}=\prod_{i=1}^{n} U_{i}
$$

For testing uniformity, the statistic $2 \ln \mathrm{Q}_{1}$ has a chi-square distribution with $2 \mathrm{n}$ degrees of freedom under the i.i.d. Uniform $(0,1)$ null hypothesis. For large values of $n, 2 \ln _{1}$ gives a uniformly most powerful test.

(c) Gini Index Test:

Let $\mathrm{X}_{1}, \mathrm{X}_{2}, \ldots, \mathrm{X}_{\mathrm{n}}$ be a random sample from an unknown distribution $\mathrm{F}$ with a probability density function $\mathrm{f}(\mathrm{x})$. Let $\mathrm{F}_{0}(\mathrm{x} ; \theta)$ be a parametric family of distributions with p.d.f. $\mathrm{f}_{0}(\mathrm{x} ; \theta)$. The hypothesis of interest is $H_{0}: f(x)=f_{0}(x ; \theta)$, for some $\theta \epsilon \Theta$, against the alternative $H_{1}: f(x) \neq f_{0}(x ; \theta)$, for any $\theta \epsilon \Theta$.

Using probability integral transformation $\mathrm{u}_{\mathrm{i}}=\mathrm{F}_{0}\left(\mathrm{x}_{\mathrm{i}}\right), \mathrm{i}=1,2, \ldots, \mathrm{n}$, it can be reduced to test the hypothesis of uniformity on the unit interval as

$\mathrm{H}_{0}: \mathrm{f}(\mathrm{u})=1,0<\mathrm{u}<1$ against the alternative $\mathrm{H}_{1}: \mathrm{f}(\mathrm{u}) \neq 1,0<\mathrm{u}<1$ and the Gini index test for testing uniformity is defined as

$$
\hat{G}_{n}=\sum_{i=1}^{n} \frac{(2 i-1) u_{(i)}}{n \sum_{i=1}^{n} u_{i}}
$$

The critical values are found in Noughabi, Arghami and Borzadaran (2014).

(d) Zhang Test:

Zhang (2002) proposed a method to construct new goodness of fit tests, derived from classical ones, and are defined as

$$
\begin{array}{r}
\mathrm{Z}_{\mathrm{A}}=-\sum_{i=1}^{n}\left[\frac{\ln \left\{U_{(i)}\right\}}{[n-i+(1 / 2)]}+\frac{\ln \left\{1-U_{(i)}\right\}}{[i-(1 / 2)]}\right] \\
\mathrm{Z}_{\mathrm{C}}=\sum_{i=1}^{n}\left[\ln \left\{\frac{U_{(i)}^{-1}-1}{[n-(1 / 2)] /[i-(3 / 4)]-1}\right\}\right]^{2}
\end{array}
$$

The statistics $\mathrm{Z}_{\mathrm{A}}$ and $\mathrm{Z}_{\mathrm{C}}$ are distribution symmetric. They appear similar to the tests KolmogorovSmirnov, Anderson-Darling and Cramer-von-Mises. The critical values for these statistics are found in Zhang (2002).

\subsubsection{Test based on sample quantiles :}

When gooodness-of-fit tests are carried out with categorized data, test statistics are constructed either with relative frequencies of intervals or with sample quantiles. In the second case, data is categorized by taking a partition of the range of $\mathrm{F}(\mathrm{x}) \in[0,1]$ and by mapping the partition to $\mathrm{R}$ with the inverse of the empirical cdf (sample quantile function). In this case, statistics are functions of the sample quantiles.

The quantile categorization is an alternative method to test goodness-of-fit, which uses the hypothetical and empirical quantile functions

$$
F_{0}^{-1}(\pi)=\inf \left\{x: F_{0}(x)>\pi\right\} \text { and } F_{n}^{-1}(\pi)=\inf \left\{x: F_{n}(x)>\pi\right\}
$$

respectively, for every $\pi$. Data are reduced by considering a partition vector of the interval $(0,1), \pi=\left(\pi_{1}, \ldots, \pi_{\mathrm{m}-1}\right)$ $\in(0,1)^{m-1}$ with

$$
\pi_{0}=0<\pi_{1}<\ldots<\pi_{\mathrm{m}-1}<1=\pi_{\mathrm{m}},
$$

and by applying the functions $F_{0}^{-1}$ and $F_{n}^{-1}$ to $\pi$ in order to obtain partition vectors of R,

$$
\begin{aligned}
& \mathrm{c}=\left(\mathrm{c}_{1}, \ldots, \mathrm{c}_{\mathrm{m}-1}\right)=\left\{F_{0}^{-1}\left(\pi_{1}\right), \ldots, F_{0}^{-1}\left(\pi_{\mathrm{m}-1}\right)\right\}, \\
& \mathrm{Y}_{\mathrm{n}}=\left(Y_{n_{1}}, \ldots, Y_{n_{m-1}}\right)=\left\{F_{n}^{-1}\left(\pi_{1}\right), \ldots, F_{n}^{-1}\left(\pi_{m-1}\right)\right\},
\end{aligned}
$$

where $Y_{n_{i}}=X_{\left(n_{i}\right)}$ and $\mathrm{n}_{\mathrm{i}}=\left(\mathrm{n} \pi_{\mathrm{i}}\right)+1, \mathrm{i}=1, \ldots, \mathrm{m}-1$. Hypothetical empirical probability vectors are

$$
q=\left(q_{1}, \ldots, q_{m}\right)=\left\{F_{0}\left(c_{j}\right)-F_{0}\left(c_{j-1}\right): 1 \leq j \leq m\right\}=\left(\pi_{j}-\pi_{j-1}: 1 \leq j \leq m\right),
$$




$$
p\left(Y_{n}\right)=\left\{p_{1}\left(Y_{n}\right), \ldots, p_{m}\left(Y_{n}\right)\right\}=\left\{F_{0}\left(Y_{n_{j}}\right)-F_{0}\left(Y_{n_{j-1}}\right): 1 \leq j \leq m\right\},
$$

where $n_{0}=0, n_{m}=\infty, Y_{n_{0}}=-\infty, Y_{n_{m}}=\infty$.

In order to test $\mathrm{H}_{0}: \mathrm{F}=\mathrm{F}_{0}$, let us consider the Cressie and Read's power divergence statistics

$$
T_{n . m}^{\lambda}=T_{n . m}^{\lambda}\left[p\left(Y_{n}\right), q\right]=\frac{2 n}{\lambda(\lambda+1)} \sum_{i=1}^{n} p_{i}\left(Y_{n}\right)\left[\left(\frac{p_{i}\left(Y_{n}\right)}{q_{i}}\right)^{\lambda}-1\right],-\infty<\lambda<\infty
$$

In case of 2 -interval partition $(0,1)=(0, \pi](\pi, 1)$, the above equation reduces to

$$
T_{n, 2, \pi}^{\lambda}=\frac{2 n}{\lambda(\lambda+1)}\left[\frac{p_{1}\left(Y_{n}\right)^{\lambda+1}}{q_{1}^{\lambda}}+\frac{\left\{1-p_{1}\left(Y_{n}\right)\right\}^{\lambda+1}}{\left(1-q_{1}\right)^{\lambda}}-1\right]
$$

Where $q_{1}=\pi$ and $p_{1}\left(Y_{n}\right)=F_{0}\left\{F_{n}^{-1}(\pi)\right\}$.

\subsubsection{The New Goodness-of-fit Test Statistic:}

To test $H_{0}: F=F_{0}$, the new statistic proposed by Esteban et al. (2006) is constructed by using the formulas

$$
S_{\max }=\sup _{\pi \in(0,1)}\left\{T_{n, 2, \pi}^{\lambda} w(\pi)\right\}, S=\int_{0}^{1} T_{n, 2, \pi}^{\lambda} d w(\pi)
$$

Where $w(s)$ is some function in the interval $[0,1]$ and large values of $S$ and $S_{\max }$ reject the null hypothesis.

Now, by taking $w(\pi)=\frac{1}{n} \pi(1-\pi)$ in $S_{\max }$, we obtain the new test statistic as follows:

$$
S_{n, A}^{1}=\max \left\{\frac{1}{n^{2}}, S_{n, A}^{1^{*}},\left(F_{0}\left(X_{(n)}\right)-1\right)^{2}\right\},
$$

Where, $S_{n, A}^{1^{*}}=\max _{i=1,2, \ldots, n-1}\left[\max \left\{\left(F_{0}\left(X_{(i)}\right)-\frac{i}{n}\right)^{2},\left(F_{0}\left(X_{(i)}\right)-\frac{i+1}{n}\right)^{2}\right\}\right]$.

\subsubsection{Types of Alternatives}

If $F(x)$ is completely specified, the $Z$, should be uniformly distributed as $U(0,1)$. Power studies hence therefore been confined to a test of the hypothesis concerning $Z$, where $Z_{i}$ 's are drawn from alternative distributions. If the variance of the hypothesized $\mathrm{F}(\mathrm{x})$ is correct, but the mean is wrong, the point $\mathrm{Z}_{\mathrm{i}}$ will tend towards 0 and 1 . Again if mean is correct, but the variance is wrong, then the point $Z_{\mathrm{i}}$ will move to each end, or will move towards 0.5 .

The following alternatives Type A, Type B and Type C proposed by Stephens (1974) are given as follows:

$$
\begin{aligned}
& A_{k}: F(z)=1-(1-z)^{k}, 0 \leq z \leq 1 \\
& B_{k}: F(z)=\left\{\begin{array}{l}
2^{k-1} z^{k}, \text { for } 0 \leq z \leq 0.5 \\
1-2^{k-1}(1-z)^{k}, \text { for } 0.5 \leq z \leq 1
\end{array}\right\} \\
& C_{k}: F(z)=\left\{\begin{array}{l}
0.5-2^{k-1}(0.5-z)^{k}, \text { for } 0 \leq z \leq 0.5 \\
0.5+2^{k-1}(z-0.5)^{k}, \text { for } 0.5 \leq z \leq 1
\end{array}\right\}
\end{aligned}
$$

where $\mathrm{k}>0$. For $\mathrm{k}>1$, the family $\mathrm{A}_{\mathrm{k}}$ gives points closer to zero than expected under the hypothesis of uniformity, $B_{k}$ gives points near to 0.5 and $C_{k}$ gives two clusters close to 0 and 1 . For $k<1$, the behavior is opposite, that is, the family $A_{k}$ gives points closer to $1, B_{k}$ gives high probability to intervals near to 0 and 1 and $C_{k}$ gives more probability to intervals around 0.5 than expected under the uniform distribution.

Also the p.d.f. of other two alternatives considered here are given as follows:

The p.d.f. of Beta distribution is

$$
f(z: \beta, \gamma)=\frac{z^{\beta-1}(1-z)^{\gamma-1}}{\int_{0}^{1} u^{\beta-1}(1-u)^{\gamma-1} d u}
$$




$$
=\frac{z^{\beta-1}(1-z)^{\gamma-1}}{B(\beta, \gamma)} ; \beta, \gamma>0,0 \leq z \leq 1
$$

The p.d.f. of Weibull distribution is

$$
f(z: \beta, \gamma)=\frac{\gamma}{\beta}\left(\frac{z}{\beta}\right)^{\gamma-1} \exp (-z / \beta)^{\gamma} ; z \geq 0, \beta, \gamma>0
$$

\section{Simulation Study}

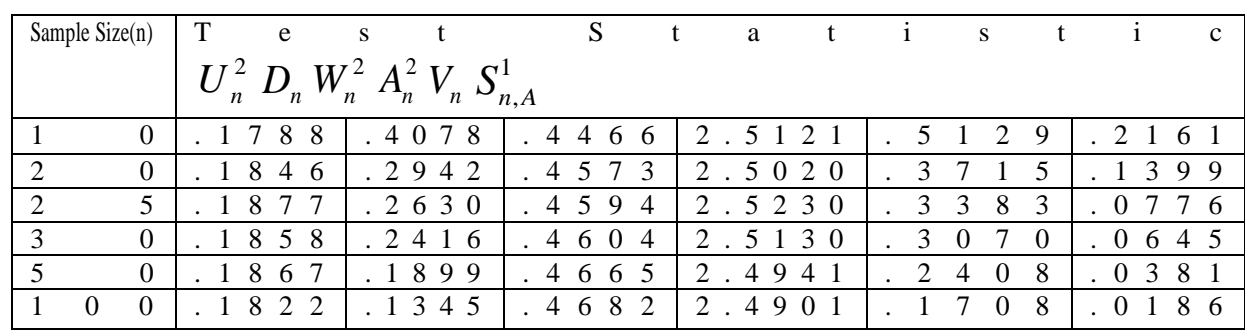

To study the empirical level and power of the eleven test statistics we have generated samples from different distributions. The study was carried out for six different sample sizes $(n=10,20,25,30,50,100)$ and considering significance levels $0.10,0.05$ and 0.01 (for 10 percent and 1 percent levels are not shown in the table) and by considering five different alternative distributions viz., Type A, Type B, Type C, Beta and Weibull. Here, the uniform variates are generated by RAND function using BASIC and for the other distributions method of inverse integral transformation is used. The ratio of the value of the test statistic greater than the critical value divided by the total number of repetition gives the empirical level under null case and power of the test statistics under the alternative hypothesis.

\section{Results}

The following tables show the simulated critical values, empirical level and power of the test statistics for different alternatives:

\section{Table1:Simulated Critical values of tests under Uniform distribution}

Table 2(a).Empirical levels of test under Uniform Distribution $(\alpha=0.05)$

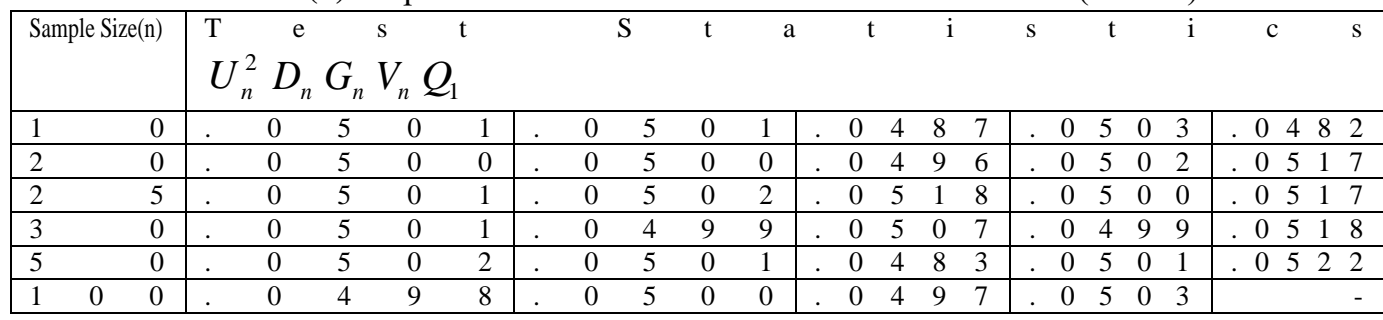

Table 2(b). Empirical levels of test under Uniform Distribution $(\alpha=0.05)$

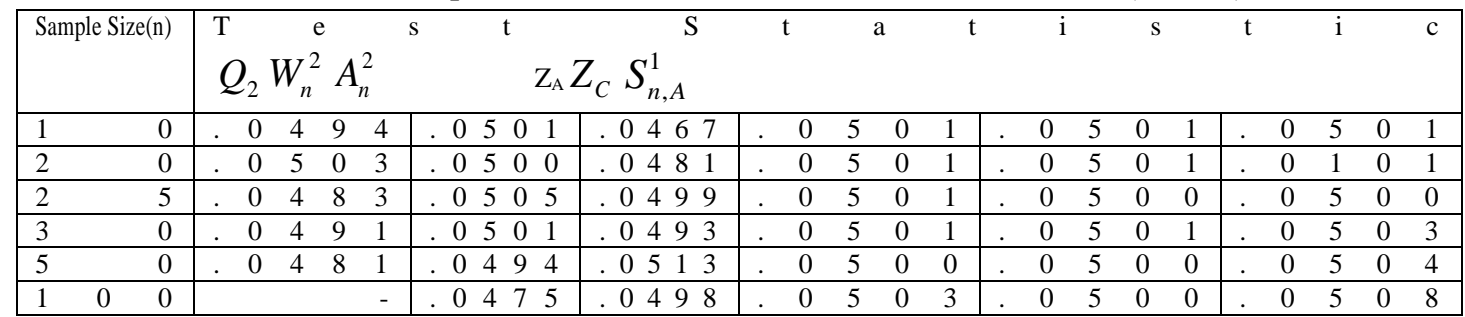

Table 3(a)Empirical Power of the Tests under Type A Distribution $(\alpha=0.05)$

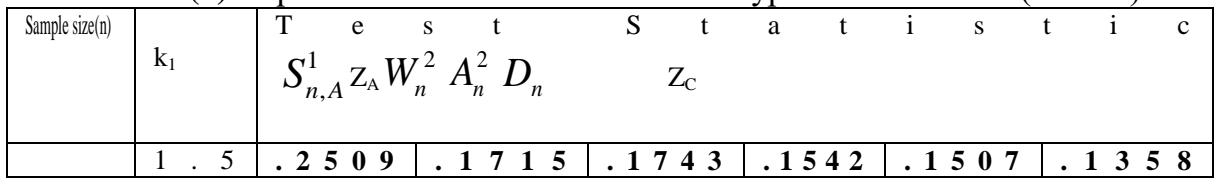


A Study of Some Tests of Uniformity and Their Performances

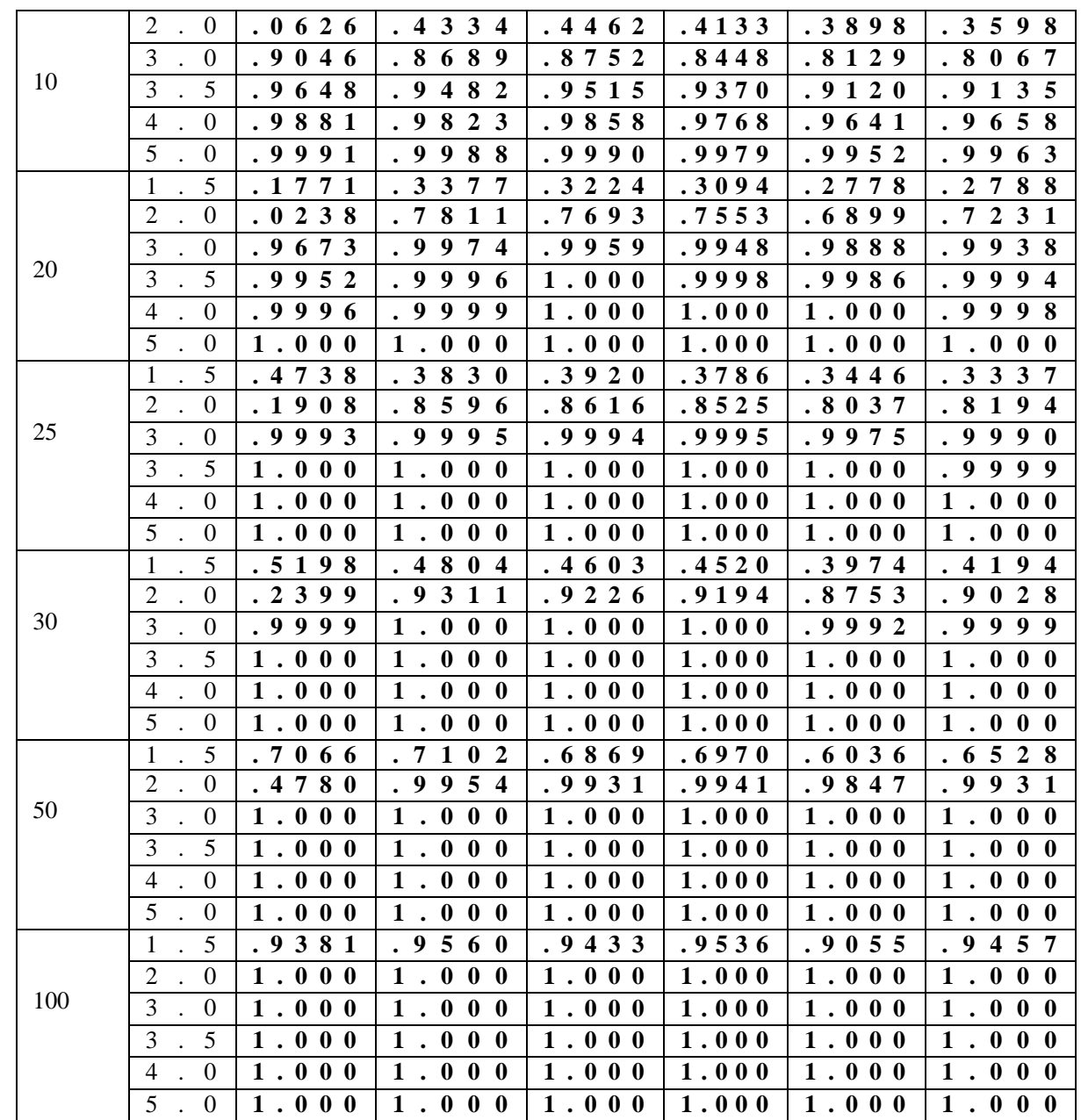

Table 3(b) Empirical Power of the Tests under Type A Distribution $(\alpha=0.05)$

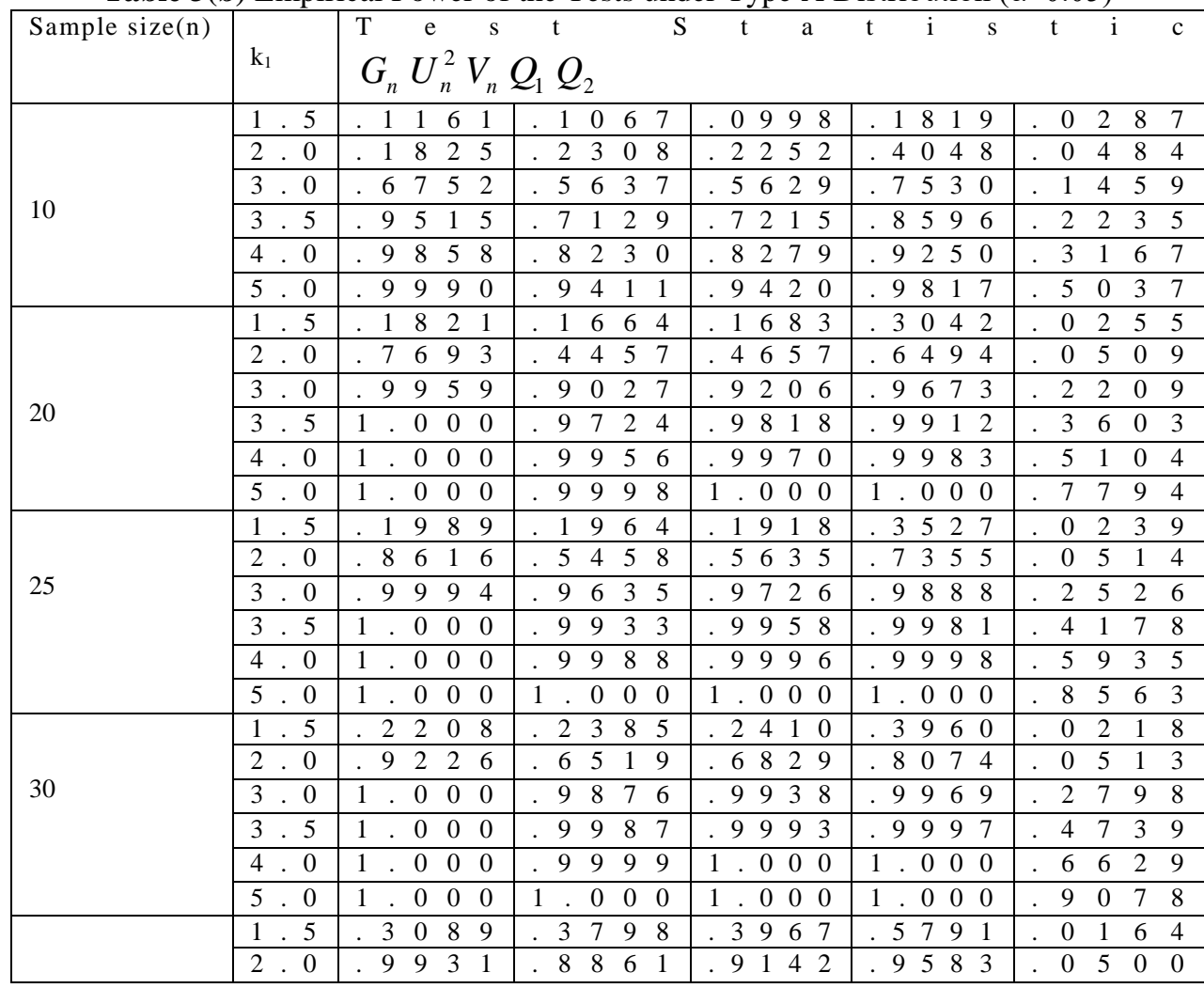


A Study of Some Tests of Uniformity and Their Performances

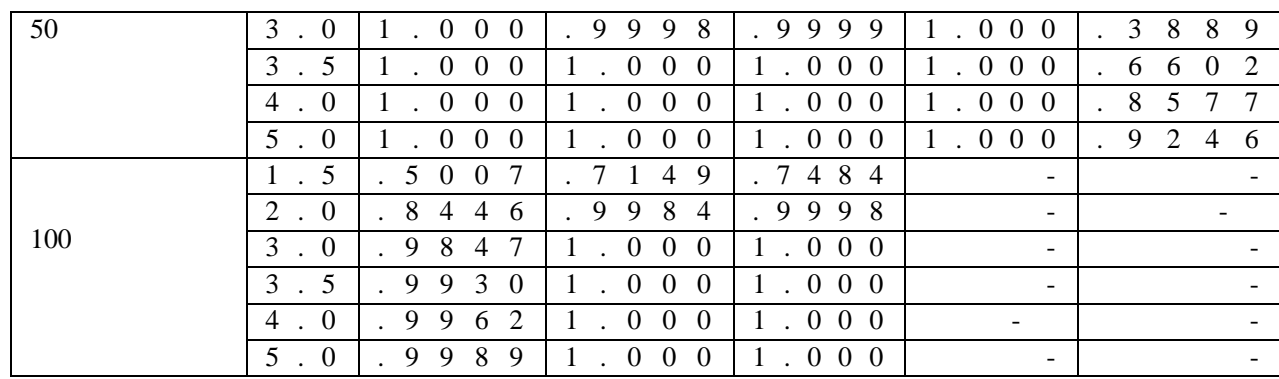

Table 4(a) Empirical Power of the Tests under Type B Distribution $(\alpha=0.05)$

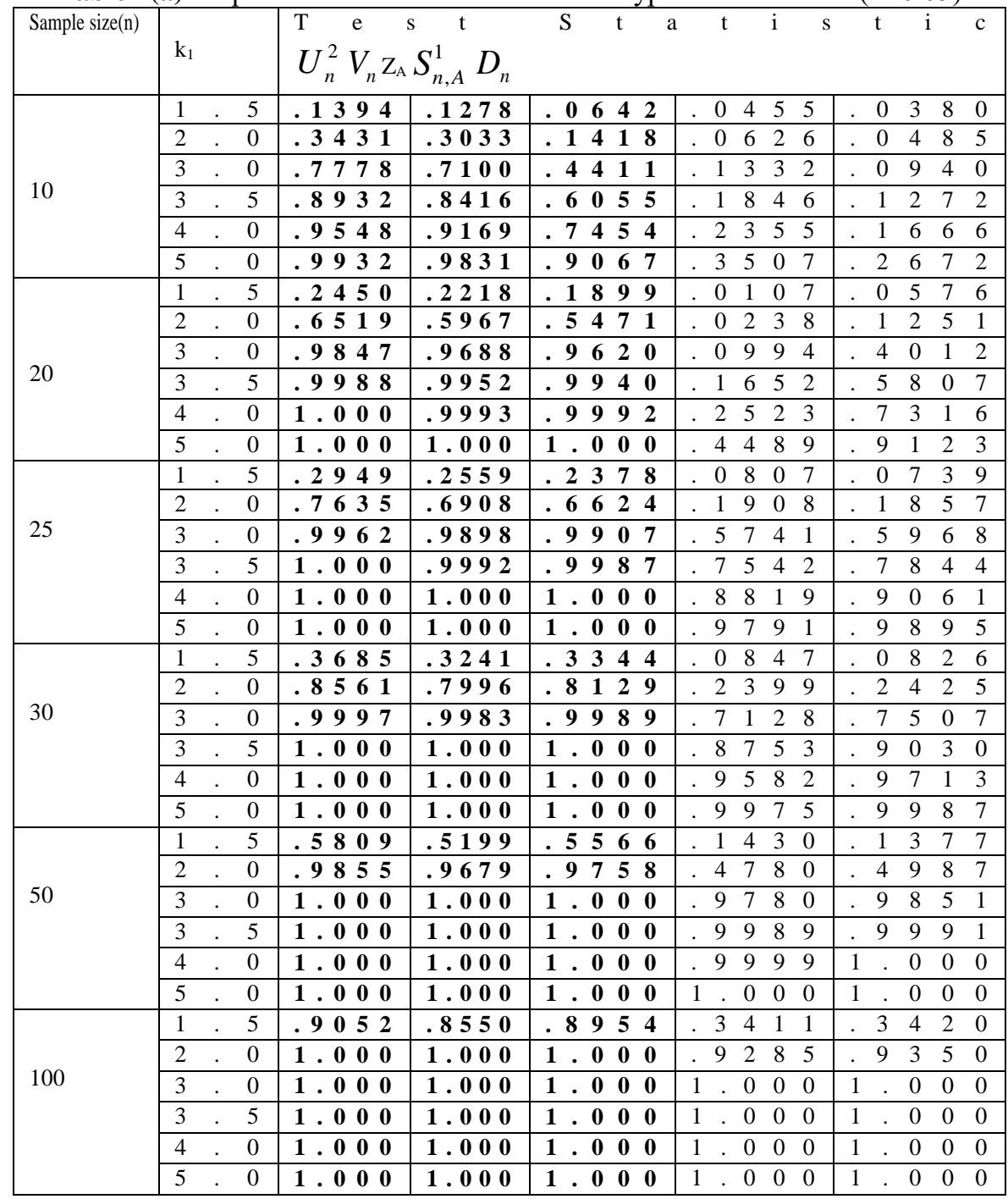

Table 4(b) Empirical Power of the Tests under Type B Distribution $(\alpha=0.05)$

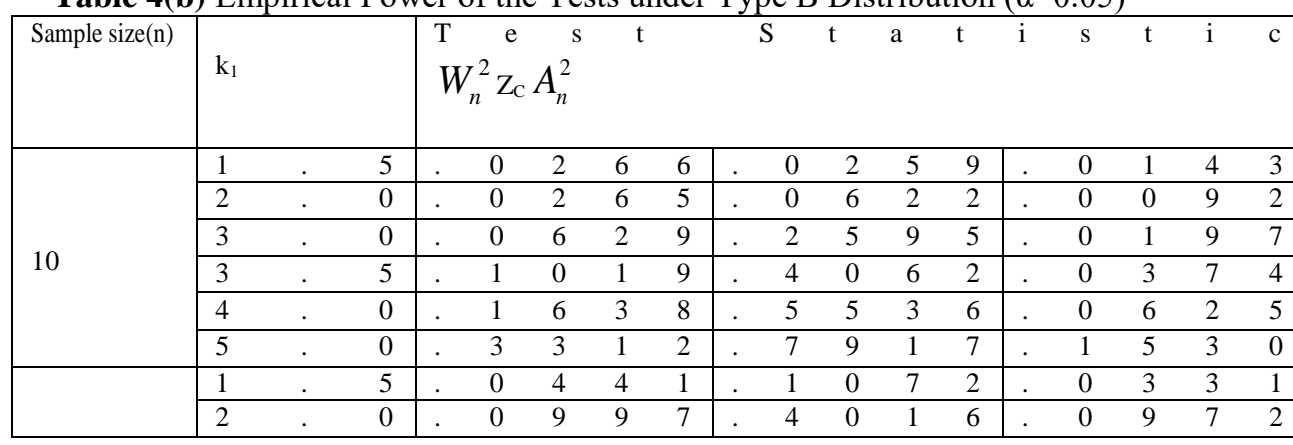


A Study of Some Tests of Uniformity and Their Performances

\begin{tabular}{|c|c|c|c|c|c|c|c|c|c|c|c|c|c|c|c|c|c|c|}
\hline \multirow{4}{*}{20} & 3 & & 0 & . & 5 & 0 & 7 & 7 & . & 9 & 2 & 6 & 1 & . & 5 & 3 & 5 & 9 \\
\hline & 3 & & 5 & . & 7 & 4 & 2 & 7 & & 9 & 8 & 6 & 2 & . & 7 & 7 & 0 & 9 \\
\hline & 4 & . & 0 & . & 8 & 8 & 9 & 9 &. & 9 & 9 & 8 & 2 & . & 9 & 0 & 8 & 7 \\
\hline & 5 & & 0 & r. & 9 & 8 & 4 & 7 & 1 & . & 0 & 0 & 0 & . & 9 & 9 & 1 & 7 \\
\hline \multirow{6}{*}{25} & 1 & . & 5 & . & 0 & 5 & 3 & 8 & 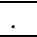 & 1 & 5 & 1 & 5 & . & 0 & 4 & 7 & 6 \\
\hline & 2 & . & 0 & . & 1 & 7 & 1 & 0 &. & 5 & 4 & 6 & 7 &. & 1 & 8 & 4 & 0 \\
\hline & 3 & . & 0 & . & 7 & 3 & 6 & 6 &. & 9 & 8 & 2 & 9 & . & 7 & 9 & 1 & 8 \\
\hline & 3 & & 5 & . & 9 & 1 & 8 & 8 & 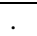 & 9 & 9 & 7 & 8 & . & 9 & 4 & 5 & 0 \\
\hline & 4 & . & 0 & . & 9 & 8 & 1 & 0 & 1 & . & 0 & 0 & 0 & . & 9 & 8 & 8 & 7 \\
\hline & 5 & 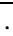 & 0 & . & 9 & 9 & 9 & 5 & 1 & . & 0 & $\overline{0}$ & 0 & 1 & . & 0 & 0 & 0 \\
\hline \multirow{6}{*}{30} & 1 & . & 5 &. & 0 & 6 & 2 & 5 &. & 2 & 1 & 8 & 4 &. & $\overline{0}$ & 6 & 0 & 5 \\
\hline & 2 & & 0 & . & 2 & 4 & 9 & 9 &. & 7 & 1 & 9 & 0 & . & 3 & 0 & 0 & 1 \\
\hline & 3 & . & 0 & . & 8 & 8 & 8 & 3 & . & 9 & 9 & 7 & 7 & . & 9 & 3 & 3 & 4 \\
\hline & 3 & & 5 & . & 9 & 7 & 8 & 8 & 1 & . & 0 & 0 & 0 & . & 9 & 9 & 1 & 4 \\
\hline & 4 & ${ }^{\circ}$ & 0 & . & 9 & 9 & 7 & 6 & 1 & . & 0 & 0 & 0 & . & 9 & 9 & 9 & 5 \\
\hline & 5 & & 0 & 1 & . & 0 & 0 & 0 & 1 & . & 0 & 0 & 0 & 1 & . & 0 & 0 & 0 \\
\hline \multirow{6}{*}{50} & 1 & & 5 & $\dot{\sigma}$ & 1 & 1 & 8 & 9 & 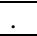 & 4 & 4 & 9 & 6 & 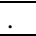 & 1 & 6 & 8 & 5 \\
\hline & 2 & & 0 & . & 6 & 1 & 2 & 3 & 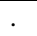 & 9 & 6 & 2 & 1 & . & 7 & 5 & 9 & 8 \\
\hline & 3 & . & 0 & . & 9 & 9 & 9 & 3 & 1 & . & 0 & 0 & 0 & . & 9 & 9 & $\overline{9}$ & 8 \\
\hline & 3 & . & 5 & 1 & . & 0 & 0 & 0 & 1 & . & 0 & 0 & 0 & 1 & . & 0 & 0 & 0 \\
\hline & 4 & . & 0 & 1 & . & 0 & 0 & 0 & 1 & . & 0 & 0 & 0 & 1 & . & 0 & 0 & 0 \\
\hline & 5 & . & 0 & 1 & . & $\overline{0}$ & 0 & 0 & 1 & . & 0 & $\overline{0}$ & 0 & 1 & . & 0 & 0 & 0 \\
\hline \multirow{6}{*}{100} & 1 & $\cdot$ & 5 & . & 3 & 7 & 9 & 5 &. & 8 & 5 & 8 & 1 & . & 5 & 6 & 5 & 5 \\
\hline & 2 & . & 0 & . & 9 & 8 & 7 & 2 &. & 9 & 9 & 9 & 9 &. & 9 & 9 & 8 & 3 \\
\hline & 3 & 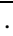 & 0 & 1 & . & 0 & 0 & 0 & 1 & . & 0 & 0 & 0 & 1 & . & 0 & 0 & 0 \\
\hline & 3 & . & 5 & 1 & . & 0 & 0 & 0 & 1 & . & 0 & 0 & 0 & 1 & . & 0 & 0 & 0 \\
\hline & 4 & . & 0 & 1 & . & 0 & 0 & 0 & 1 & . & 0 & 0 & 0 & 1 & . & 0 & 0 & 0 \\
\hline & 5 & & 0 & 1 & . & 0 & 0 & 0 & 1 & . & 0 & 0 & 0 & 1 & . & 0 & 0 & 0 \\
\hline
\end{tabular}

Table 5(a) Empirical Power of the Tests under Type C Distribution $(\alpha=0.05)$

\begin{tabular}{|c|c|c|c|c|c|c|c|c|c|c|c|c|c|c|}
\hline \multirow{2}{*}{ Sample size(n) } & \multicolumn{2}{|c|}{$\mathrm{k}_{1}$} & \multicolumn{12}{|c|}{$U_{n}^{2} V_{n} Q_{2} A_{n}^{2} D_{n} S_{n, A}^{1}$} \\
\hline & 1 & 5 & .1389 & .1253 & .1868 & 1 & 1 & 6 & 8 & .11105 & 1 & 0 & 0 & 2 \\
\hline \multirow{5}{*}{10} & 2 & 0 & .3429 & .3036 & .383 & .2 & 2 & 1 & 7 & .1956 & 1 & 6 & 5 & 9 \\
\hline & 3 & 0 & .7745 & .7104 & .7587 & 4 & 9 & $\mathbf{1}$ & 4 & .3701 & 3 & 0 & 2 & 7 \\
\hline & 3 & 5 & .8905 & .8400 & .8622 & .6 & 2 & 4 & 8 & .4514 & 3 & 5 & 9 & 3 \\
\hline & 4 & 0 & .95514 & .9177 & .9258 & . 7 & 3 & 9 & $\mathbf{0}$ & .5258 & 4 & 0 & 9 & 0 \\
\hline & 5 & 0 & .9927 & .9805 & .9808 & .8 & 9 & 2 & 9 & .6434 & 4 & 9 & 4 & 2 \\
\hline \multirow{6}{*}{20} & 1 & 5 & .2495 & .2290 & .306 & . 1 & 5 & 2 & 5 & .1480 & 0 & 4 & 6 & 2 \\
\hline & 2 & 0 & .65567 & .5992 & .6495 & .3 & 7 & $\mathbf{3}$ & 1 & .3106 & 1 & 1 & 3 & 6 \\
\hline & 3 & 0 & .9863 & .9710 & .9642 & .8 & 4 & $\mathbf{3}$ & 7 & .6521 & 2 & 8 & 7 & 4 \\
\hline & 3 & 5 & .9985 & .9952 & .9919 & . 9 & 4 & 5 & 4 & .7842 & 3 & 7 & 6 & 6 \\
\hline & 4 & 0 & 1.000 & .9995 & .9987 & .9 & 8 & 4 & 7 & .8770 & 4 & 6 & 1 & 0 \\
\hline & 5 & 0 & 1.000 & 1.000 & 1.000 & . 9 & 9 & 9 & 3 & .9674 & 6 & 1 & 8 & 1 \\
\hline \multirow{6}{*}{25} & 1 & 5 & .2969 & .2604 & .358 & . 1 & 7 & 1 & 4 & .17721 & 1 & 6 & 1 & 6 \\
\hline & 2 & 0 & .7639 & .6940 & .739 & .4 & 5 & 1 & 9 & .3862 & 3 & 4 & 2 & 8 \\
\hline & 3 & 0 & .9963 & .9909 & .986 & . 9 & 2 & 7 & 1 & .7858 & 7 & 1 & 6 & 8 \\
\hline & 3 & 5 & .9998 & .9991 & .9977 & .9 & 8 & $\mathbf{3}$ & 7 & .8976 & 8 & 4 & 0 & 4 \\
\hline & 4 & 0 & 1.000 & 1.000 & .9999 & .9 & 9 & 7 & 4 & .9567 & 9 & 2 & 3 & 4 \\
\hline & 5 & 0 & 1.000 & 1.000 & 1.000 & 1. & $\mathbf{0}$ & $\mathbf{0}$ & $\mathbf{0}$ & .9948 & 9 & 8 & 5 & 5 \\
\hline \multirow{6}{*}{30} & 1 & 5 & .3648 & .3265 & .4029 & . 1 & 8 & 8 & 7 & .1862 & 1 & 7 & 4 & 9 \\
\hline & 2 & 0 & .885333 & .7994 & .8095 & . 5 & 3 & $\mathbf{3}$ & 5 & .4408 & 3 & $\overline{9}$ & 6 & 8 \\
\hline & 3 & 0 & .9994 & .9984 & .9958 & . 9 & 7 & 2 & 2 & .8702 & 8 & 1 & 3 & 2 \\
\hline & 3 & 5 & 1.000 & .9999 & .9998 & . 9 & 9 & 7 & 2 & .9550 & 9 & 2 & 3 & 3 \\
\hline & 4 & 0 & 1.000 & 1.000 & 1.000 & . 9 & 9 & 9 & 7 & .9879 & 9 & 7 & 2 & 8 \\
\hline & 5 & 0 & 1.000 & 1.000 & 1.00 & 1. & $\mathbf{0}$ & $\mathbf{0}$ & $\mathbf{0}$ & .9994 & 9 & 9 & 7 & 6 \\
\hline \multirow{7}{*}{50} & 1 & 5 & .55833 & .5205 & .5821 & .2 & $\overline{8}$ & 9 & 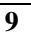 & .2592 & 2 & 4 & 8 & 1 \\
\hline & 2 & 0 & .9799 & .9625 & .9460 & .8 & 1 & $\mathbf{0}$ & 2 & .6497 & 6 & 1 & 5 & 8 \\
\hline & 3 & 0 & 1.000 & 1.000 & & .9 & 9 & 9 & 6 & .999110 & 9 & 8 & 7 & 9 \\
\hline & 3 & 5 & 1.000 & 1.000 & & 1. & 0 & $\mathbf{0}$ & 0 & .9997 & 9 & 9 & 9 & 1 \\
\hline & 4 & 0 & 1.000 & 1.000 & & 1. & 0 & $\mathbf{0}$ & 0 & $\begin{array}{llllll}1 . & 0 & 0 & 0\end{array}$ & 1 & 0 & 0 & 0 \\
\hline & 5 & 0 & 1.000 & 1.000 & & 1. & $\mathbf{0}$ & $\mathbf{0}$ & 0 & $\begin{array}{lllll}1 . & 0 & 0 & 0\end{array}$ & 1. & 0 & 0 & 0 \\
\hline & & 5 & .9033 & .8576 & & .5 & & 6 & & .4817 & & & & 6 \\
\hline
\end{tabular}


A Study of Some Tests of Uniformity and Their Performances

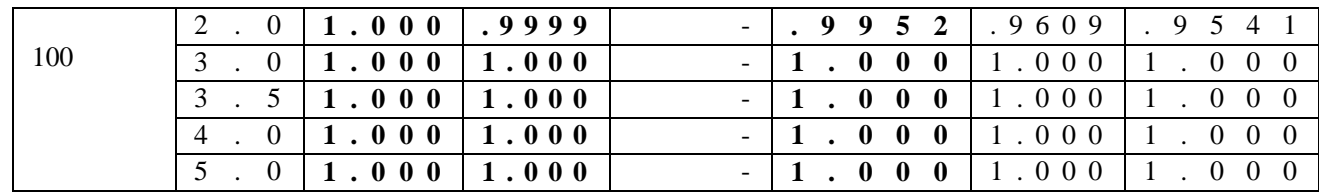

Table 5(b) Empirical Power of the Tests under Type C Distribution ( $\alpha=0.05)$

\begin{tabular}{|c|c|c|c|c|c|c|c|c|c|c|c|c|c|c|c|c|c|c|c|c|c|c|c|}
\hline Sample size(n) & $\mathrm{k}_{1}$ & $\begin{array}{l}\mathrm{T} \\
W_{n}^{2}\end{array}$ & & & & & & & & $\mathrm{~S}$ & & & $a$ & & $\mathrm{t}$ & & & s & & & i & & c \\
\hline \multirow{6}{*}{10} & 1.5 & 0 & & 7 & & & 0 & 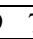 & & 3 & & 0 & 3 & 9 & 8 & 0 & 6 & 7 & 7 & & 0 & & 9 \\
\hline & 2.0 & .1 & 5 & 5 & & & 13 & ; & 5 & 4 & & 1 & 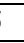 & 4 & 7 & .1 & 1 & 7 & 5 & 1 & 7 & 0 & 1 \\
\hline & 3.0 & .2 & 9 & 9 & & 2 & 2 & 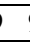 & 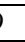 & 7 & & 3 & 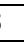 & 4 & 3 & .2 & 7 & 5 & 0 & 2 & 8 & 7 & 6 \\
\hline & 3.5 & .3 & 7 & 9 & & & 37 & 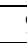 & 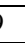 & 3 & & 4 & 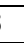 & 5 & 5 & .3 & 7 & 2 & 9 & & 4 & 2 & 2 \\
\hline & 4.0 & .4 & 6 & 0 & & 4 & 4 & - & ) & 5 & & 5 & 3 & 7 & 2 & 4 & 7 & 2 & 6 & 3 & 8 & 3 & 0 \\
\hline & 5.0 & .6 & 1 & 9 & & & 53 & 3 & ) & 7 & & 7 & 2 & 4 & 1 & 6 & 6 & 7 & 9 & & 5 & 3 & 6 \\
\hline \multirow{6}{*}{20} & 1.5 & .1 & & 6 & & & 1 & - & 5 & 5 & & 1 & 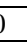 & 3 & 9 & .0 & 7 & 0 & 5 & & 3 & 7 & 2 \\
\hline & 2.0 & .2 & 4 & 4 & & 2 & 22 & + & + & 2 & & 2 & 3 & 4 & 6 & .1 & 7 & 6 & 8 & 2 & 3 & 9 & 6 \\
\hline & 3.0 & .6 & 6 & 8 & & 6 & 5 & 5 & 3 & 8 & . & 6 & 2 & 8 & 0 & .5 & 6 & 2 & 6 & 4 & 1 & 1 & 3 \\
\hline & 3.5 & 8 & 4 & 2 & & 8 & 8 & t & 2 & 1 & & 7 & 9 & 0 & 2 & .7 & 4 & 2 & 9 & 4 & 7 & 7 & 1 \\
\hline & 4.0 & .9 & 3 & 7 & & .9 & 93 & 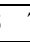 & 7 & & & 8 & 9 & 6 & 8 & .8 & 6 & 3 & 6 & 5 & 3 & 5 & 0 \\
\hline & 5.0 & .9 & 9 & 4 & & & 67 & 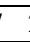 & 2 & 0 & & 9 & 3 & 2 & 1 & .9 & 7 & 4 & 3 & 6 & 3 & 1 & 1 \\
\hline \multirow{6}{*}{25} & 1.5 & .1 & 2 & 8 & & .1 & 12 & 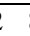 & 3 & 8 & & 1 & 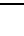 & 2 & 5 & .0 & 7 & 0 & 9 & 1 & 4 & 9 & 4 \\
\hline & 2.0 & .3 & 1 & 3 & & .3 & 3 & 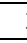 & 3 & 1 & & 2 & 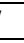 & 0 & 0 & .1 & 8 & 7 & 3 & 2 & 6 & 4 & 5 \\
\hline & 3.0 & .8 & 3 & 0 & & .8 & 83 & 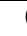 & ) & 7 & & 7 & & 8 & 2 & .6 & 5 & 7 & 6 & 4 & 5 & 9 & 4 \\
\hline & 3.5 & .9 & 4 & 7 & & .9 & 92 & 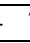 & 7 & 5 & & 8 & 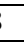 & 8 & 7 & .8 & 3 & 8 & 2 & 5 & 3 & 8 & 7 \\
\hline & 4.0 & .9 & 8 & 8 & & .9 & 98 & 3 & 3 & 3 & & 9 & & 0 & 7 & .9 & 3 & 5 & & 6 & 0 & 4 & 0 \\
\hline & 5.0 & .9 & 9 & 9 & & .7 & 7 & 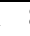 & 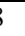 & 5 & & 9 & & 6 & 1 & .9 & 9 & 2 & 0 & 6 & 9 & 6 & 6 \\
\hline \multirow{6}{*}{30} & 1.5 & .1 & 3 & 7 & & & 1 & & 7 & 9 & & 1 & 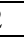 & 2 & 0 & .0 & 8 & 5 & 2 & 1 & 5 & 7 & 8 \\
\hline & 2.0 & . 3 & 8 & 4 & & & 3 & 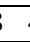 & 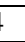 & 5 & & 3 & & 0 & 2 & . 2 & 6 & 5 & 7 & 2 & 8 & 8 & 1 \\
\hline & 3.0 & .9 & 2 & 5 & & 9 & 9 & 2 & 5 & 1 & & 8 & & 3 & 8 & 8 & 0 & 6 & 3 & 5 & 0 & 7 & 6 \\
\hline & 3.5 & .9 & 8 & 7 & & 9 & 9 & 3 & 7 & 7 & & 9 & 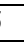 & 5 & 4 & .9 & 3 & 8 & 6 & 5 & 8 & 8 & 5 \\
\hline & 4.0 & .9 & 9 & 8 & & & 7 & 2 & 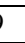 & 5 & & 9 & 3 & 9 & 0 & .9 & 8 & 4 & 6 & 6 & 5 & 6 & 3 \\
\hline & 5.0 & 1 & 0 & 0 & & 7 & 7 & 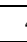 & 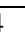 & 9 & & 9 & 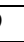 & 9 & 6 & 9 & 9 & 9 & 4 & 7 & 5 & 0 & 8 \\
\hline \multirow{6}{*}{50} & 1.5 & .2 & & 4 & & & 2 & 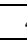 & 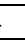 & 9 & & 1 & 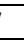 & 0 & 2 & .1 & 1 & 8 & 7 & & 9 & 3 & 3 \\
\hline & 2.0 & .6 & 8 & 6 & & 5 & 5 & 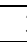 & 3 & 8 & & 5 & 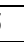 & 3 & 8 & . 4 & 7 & 8 & 5 & & 7 & 3 & 8 \\
\hline & 3.0 & . 9 & 9 & 8 & & .7 & 7 & 5 & 3 & 4 & & 9 & 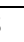 & 8 & 6 & . 9 & 8 & 3 & 1 & & & & - \\
\hline & 3.5 & 1 & 0 & 0 & & 1 & . & & ) & j & & 9 & & 9 & 0 & .9 & 9 & 8 & 5 & & & & - \\
\hline & 4.0 & 1 & 0 & 0 & & 1 & . & & ) & 5 & 1 & . & ) & 0 & 0 & .9 & 9 & 9 & 9 & & & & - \\
\hline & 5.0 & 1 & 0 & 0 & & 1 & 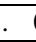 & 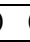 & 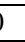 & ) & 1 & . & ) & 0 & 0 & 1. & 0 & 0 & 0 & & & & - \\
\hline \multirow{6}{*}{100} & 1.5 & .4 & 5 & 1 & & .5 & 5 & 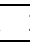 & 3 & 1 & & 3 & 5 & 2 & 2 & .2 & 8 & 1 & & & & & - \\
\hline & 2.0 & .9 & 8 & 9 & & 8 & 8 & 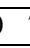 & 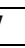 & 3 & & 9 & 3 & 0 & 6 & .9 & 0 & 8 & 4 & & & & - \\
\hline & 3.0 & 1 & 0 & 0 & & 9 & 9 & 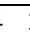 & 3 & 5 & 1 & . & & 0 & 0 & 1 & 0 & 0 & 0 & & & & - \\
\hline & 3.5 & 1 & 0 & 0 & & 9 & 9 & & 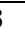 & 5 & 1 & & & 0 & 0 & 1 & 0 & 0 & 0 & & & & - \\
\hline & 4.0 & 1 & 0 & 0 & & 9 & 9 & & 2 & 5 & 1 & & 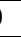 & 0 & 0 & 1 & 0 & 0 & 0 & & & & - \\
\hline & 5.0 & 1 & & 0 & & 9 & 9 & 3 & 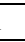 & 6 & 1 & & 0 & 0 & 0 & 1 & 0 & 0 & 0 & & & & - \\
\hline
\end{tabular}

Table 6(a) Empirical Power of the Tests under Beta Distribution $(\alpha=0.05)$

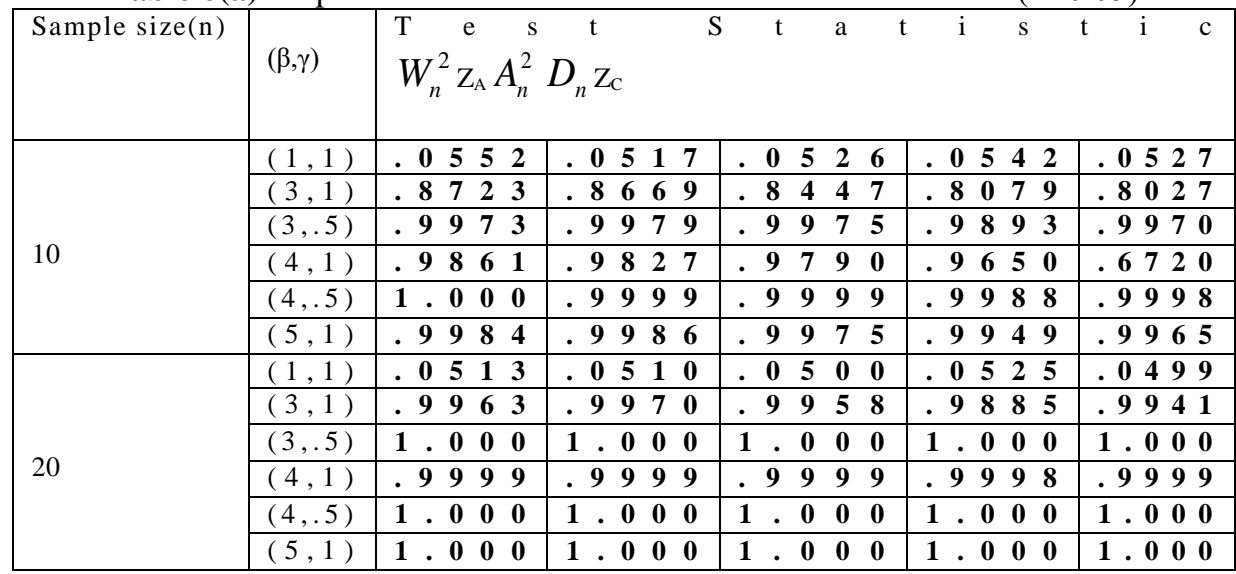


A Study of Some Tests of Uniformity and Their Performances

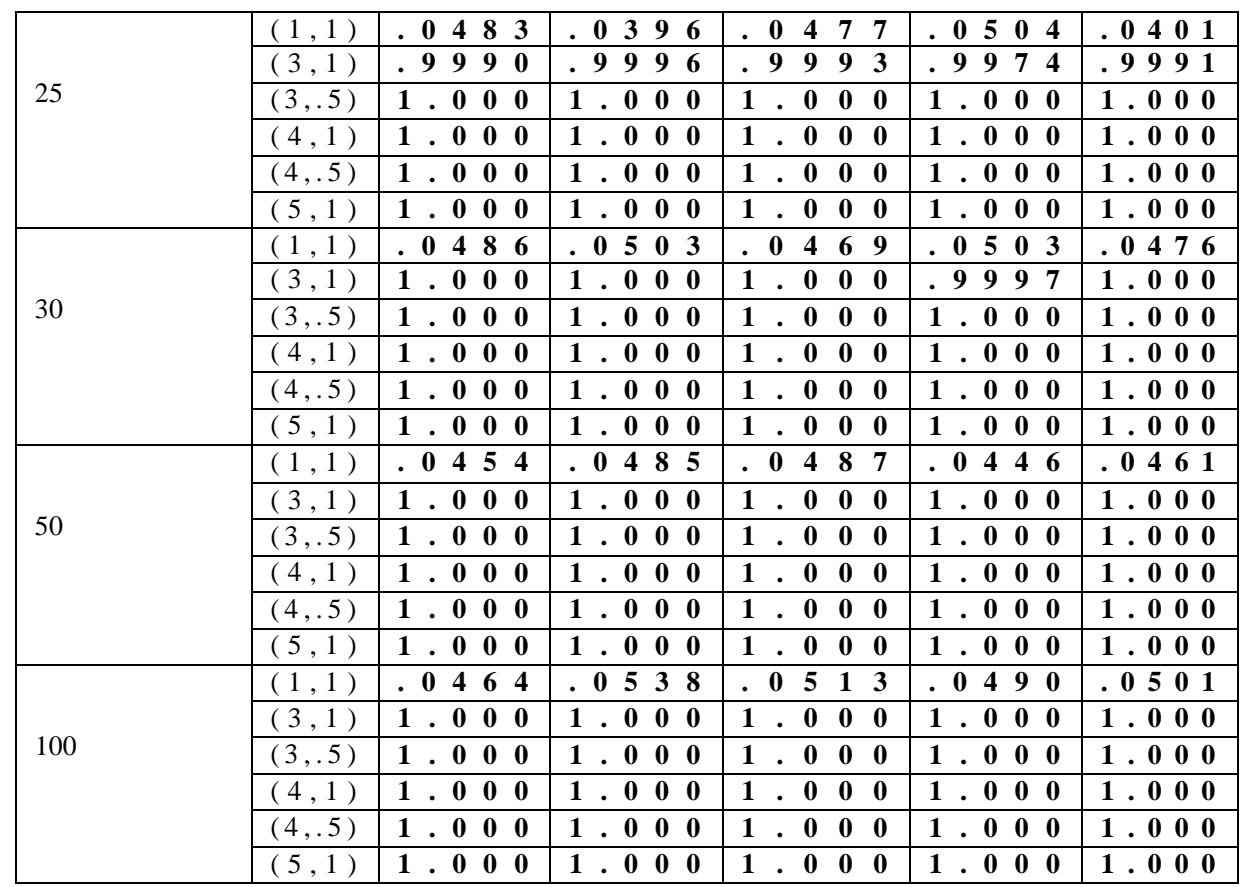

Table 6(b) Empirical Power of the Tests under Beta Distribution $(\alpha=0.05)$

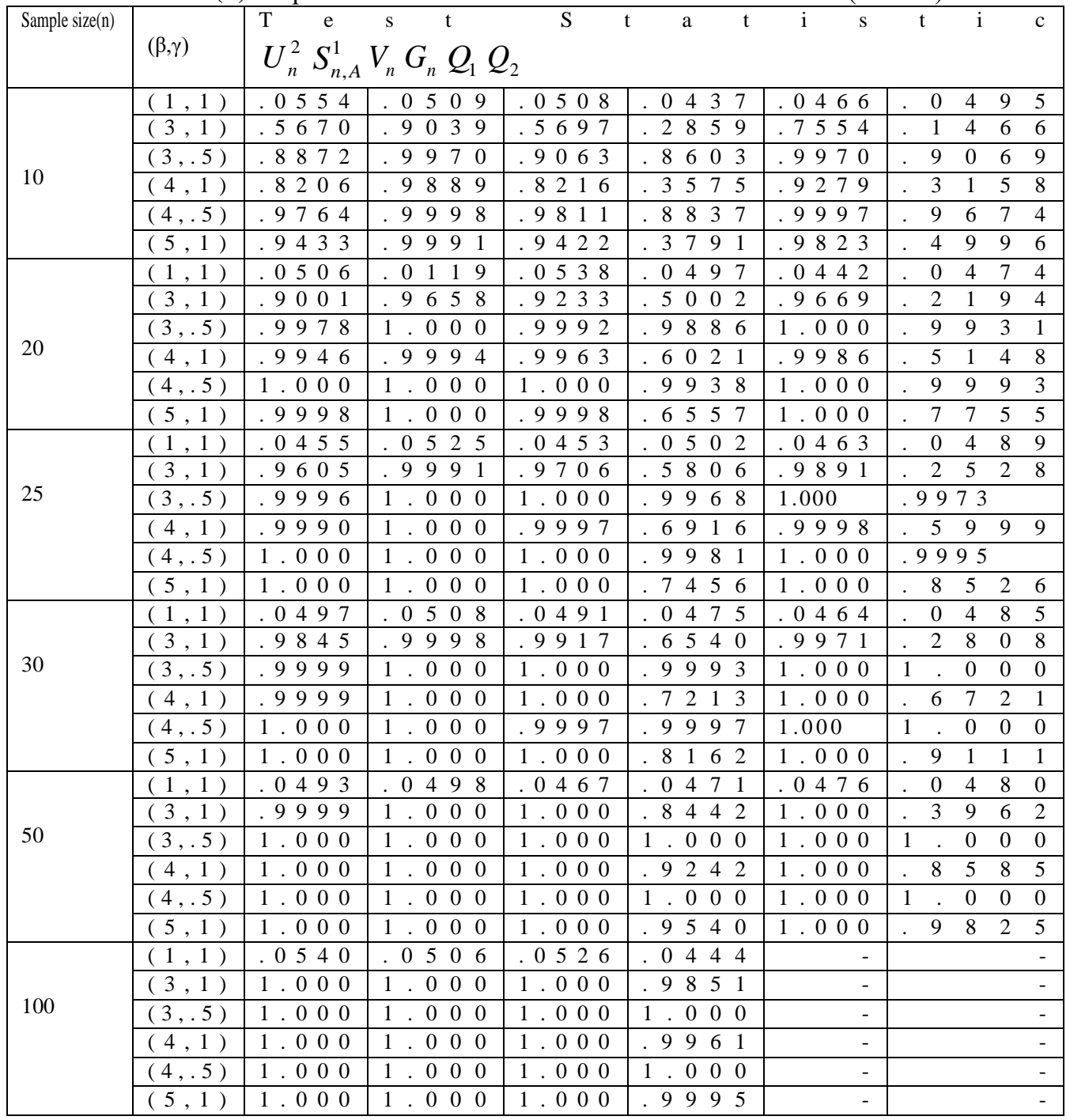


Table 7(a) Empirical Power of the Tests under Weibull Distribution $(\alpha=0.05)$

\begin{tabular}{|c|c|c|c|c|c|c|c|c|c|c|c|c|c|c|c|c|c|c|c|c|c|}
\hline Sample size(n) & $(\beta, \gamma)$ & & V & & & & \multicolumn{2}{|c|}{$\mathrm{t}$} & & & $\mathrm{t}$ & \multicolumn{2}{|c|}{$\mathrm{a}$} & $\mathrm{t}$ & i & \multicolumn{2}{|c|}{$\mathrm{s}$} & $t$ & \multicolumn{2}{|c|}{ i } & c \\
\hline \multirow{6}{*}{10} & $(1,1)$ & . & 9 & 9 & 1 & 4 & . & 9 & 7 & 0 & 1 & . & 9 & 6 & 8 & 2 & . & 9 & 6 & 6 & 6 \\
\hline & $(3,1)$ & 1 & . & $\mathbf{0}$ & 0 & 0 & . & 9 & 9 & 9 & 9 & . & 9 & 9 & 9 & 9 & . & 9 & 9 & 9 & 9 \\
\hline & $(3, .5)$ & 1 & . & $\mathbf{0}$ & 0 & 0 & . & 9 & 9 & 9 & 8 & . & 9 & 9 & 9 & 8 & . & 9 & 9 & 9 & 6 \\
\hline & $(4,1)$ & 1 & . & 0 & 0 & 0 & 1 & . & 0 & 0 & 0 & 1 & . & $\mathbf{0}$ & 0 & $\mathbf{0}$ & 1 & . & $\mathbf{0}$ & 0 & $\mathbf{0}$ \\
\hline & $(4, .5)$ & 1 & . & $\mathbf{0}$ & $\mathbf{0}$ & 0 & . & 9 & 9 & 9 & 8 & . & 9 & 9 & 9 & 9 & 1 & . & $\mathbf{0}$ & $\mathbf{0}$ & $\mathbf{0}$ \\
\hline & $(5,1)$ & 1 & . & $\mathbf{0}$ & $\mathbf{0}$ & 0 & 1 & • & 0 & 0 & 0 & 1 & • & 0 & 0 & $\mathbf{0}$ & $\mathbf{1}$ & . & 0 & 0 & 0 \\
\hline \multirow{6}{*}{20} & $(1,1)$ & . & 9 & 9 & 9 & 8 & . & 9 & 9 & 9 & 5 & . & 9 & 9 & 9 & 3 & . & 9 & 9 & 9 & 4 \\
\hline & $(3,1)$ & 1 & . & $\mathbf{0}$ & 0 & $\mathbf{0}$ & 1 & . & $\mathbf{0}$ & 0 & $\mathbf{0}$ & 1 & . & $\mathbf{0}$ & $\mathbf{0}$ & $\mathbf{0}$ & 1 & . & $\mathbf{0}$ & $\mathbf{0}$ & $\mathbf{0}$ \\
\hline & $(3, .5)$ & 1 & . & $\mathbf{0}$ & $\mathbf{0}$ & 0 & 1 & . & 0 & $\mathbf{0}$ & 0 & 1 & . & $\mathbf{0}$ & $\mathbf{0}$ & $\mathbf{0}$ & 1 & . & $\mathbf{0}$ & $\mathbf{0}$ & $\mathbf{0}$ \\
\hline & $(4,1)$ & 1 & . & $\mathbf{0}$ & $\mathbf{0}$ & $\mathbf{0}$ & 1 & . & $\mathbf{0}$ & $\mathbf{0}$ & $\mathbf{0}$ & 1 & . & $\mathbf{0}$ & $\mathbf{0}$ & $\mathbf{0}$ & 1 & . & $\mathbf{0}$ & 0 & $\mathbf{0}$ \\
\hline & $(4, .5)$ & 1 & . & $\overline{0}$ & $\overline{0}$ & 0 & 1 & . & 0 & 0 & 0 & 1 & . & $\mathbf{0}$ & 0 & $\mathbf{0}$ & 1 & . & 0 & 0 & $\mathbf{0}$ \\
\hline & $(5,1)$ & 1 & . & 0 & 0 & 0 & 1 & . & 0 & 0 & 0 & 1 & • & 0 & 0 & $\mathbf{0}$ & 1 & . & 0 & 0 & 0 \\
\hline \multirow{6}{*}{25} & $(1,1)$ & 1 & . & $\mathbf{0}$ & 0 & 0 & 1 & . & $\mathbf{0}$ & 0 & $\mathbf{0}$ & - & 9 & 9 & 9 & 9 & . & 9 & 9 & 9 & 8 \\
\hline & $(3,1)$ & 1 & . & $\mathbf{0}$ & $\mathbf{0}$ & $\mathbf{0}$ & 1 & . & $\mathbf{0}$ & $\mathbf{0}$ & $\mathbf{0}$ & 1 & . & $\mathbf{0}$ & $\mathbf{0}$ & $\mathbf{0}$ & 1 & . & $\mathbf{0}$ & $\mathbf{0}$ & $\mathbf{0}$ \\
\hline & $(3, .5)$ & 1 & . & $\mathbf{0}$ & $\mathbf{0}$ & $\mathbf{0}$ & 1 & - & $\mathbf{0}$ & $\mathbf{0}$ & 0 & 1 & . & $\mathbf{0}$ & $\mathbf{0}$ & $\mathbf{0}$ & 1 & . & $\mathbf{0}$ & $\mathbf{0}$ & $\mathbf{0}$ \\
\hline & $(4,1)$ & 1 & . & $\mathbf{0}$ & 0 & 0 & 1 & . & 0 & 0 & 0 & 1 & . & 0 & $\mathbf{0}$ & $\mathbf{0}$ & 1 & . & 0 & $\mathbf{0}$ & $\mathbf{0}$ \\
\hline & $(4, .5)$ & 1 & . & $\mathbf{0}$ & 0 & 0 & 1 & . & 0 & 0 & 0 & 1 & . & $\mathbf{0}$ & 0 & 0 & 1 & . & 0 & 0 & $\mathbf{0}$ \\
\hline & $(5,1)$ & 1 & . & $\mathbf{0}$ & $\mathbf{0}$ & 0 & 1 & . & $\mathbf{0}$ & 0 & $\mathbf{0}$ & 1 & . & $\mathbf{0}$ & $\mathbf{0}$ & $\mathbf{0}$ & 1 & . & 0 & $\mathbf{0}$ & $\mathbf{0}$ \\
\hline \multirow{6}{*}{30} & $(1,1)$ & 1 & . & $\mathbf{0}$ & $\mathbf{0}$ & 0 & 1 & . & 0 & $\mathbf{0}$ & 0 & 1 & . & $\mathbf{0}$ & 0 & $\mathbf{0}$ & 1 & . & 0 & $\mathbf{0}$ & $\mathbf{0}$ \\
\hline & $(3,1)$ & 1 & . & $\mathbf{0}$ & $\mathbf{0}$ & $\mathbf{0}$ & 1 & . & 0 & 0 & $\mathbf{0}$ & 1 & . & $\mathbf{0}$ & $\mathbf{0}$ & $\mathbf{0}$ & 1 & . & 0 & $\mathbf{0}$ & $\mathbf{0}$ \\
\hline & $(3, .5)$ & 1 & . & $\mathbf{0}$ & 0 & 0 & 1 & . & 0 & 0 & 0 & 1 & . & 0 & 0 & $\mathbf{0}$ & 1 & . & 0 & 0 & $\mathbf{0}$ \\
\hline & $(4,1)$ & 1 & . & $\mathbf{0}$ & $\mathbf{0}$ & $\mathbf{0}$ & 1 & . & $\mathbf{0}$ & 0 & $\mathbf{0}$ & 1 & . & $\mathbf{0}$ & $\mathbf{0}$ & $\mathbf{0}$ & 1 & . & 0 & $\mathbf{0}$ & $\mathbf{0}$ \\
\hline & $(4, .5)$ & 1 & . & $\mathbf{0}$ & $\overline{0}$ & 0 & 1 & . & 0 & 0 & 0 & 1 & . & $\mathbf{0}$ & 0 & $\mathbf{0}$ & 1 & . & 0 & 0 & $\mathbf{0}$ \\
\hline & $(5,1)$ & 1 & . & $\mathbf{0}$ & $\mathbf{0}$ & 0 & 1 & . & $\mathbf{0}$ & 0 & $\mathbf{0}$ & 1 & . & $\mathbf{0}$ & $\mathbf{0}$ & $\mathbf{0}$ & 1 & . & 0 & $\mathbf{0}$ & $\mathbf{0}$ \\
\hline \multirow{6}{*}{50} & $(1,1)$ & 1 & . & $\mathbf{0}$ & $\mathbf{0}$ & 0 & 1 & . & 0 & $\mathbf{0}$ & 0 & 1 & . & $\mathbf{0}$ & 0 & $\mathbf{0}$ & 1 & . & 0 & $\mathbf{0}$ & $\mathbf{0}$ \\
\hline & $(3,1)$ & 1 & . & $\mathbf{0}$ & $\mathbf{0}$ & $\mathbf{0}$ & 1 & . & 0 & 0 & $\mathbf{0}$ & 1 & . & $\mathbf{0}$ & $\mathbf{0}$ & $\mathbf{0}$ & 1 & . & 0 & $\mathbf{0}$ & $\mathbf{0}$ \\
\hline & $(3, .5)$ & 1 & . & $\mathbf{0}$ & $\overline{0}$ & 0 & 1 & . & 0 & 0 & 0 & 1 & . & 0 & 0 & $\mathbf{0}$ & 1 & . & 0 & 0 & $\mathbf{0}$ \\
\hline & $(4,1)$ & 1 & . & 0 & $\mathbf{0}$ & 0 & 1 & . & 0 & 0 & $\mathbf{0}$ & 1 & . & $\mathbf{0}$ & $\mathbf{0}$ & $\mathbf{0}$ & 1 & . & 0 & 0 & $\mathbf{0}$ \\
\hline & $(4, .5)$ & 1 & . & $\mathbf{0}$ & $\mathbf{0}$ & $\mathbf{0}$ & 1 & . & 0 & $\mathbf{0}$ & $\mathbf{0}$ & 1 & . & $\mathbf{0}$ & 0 & $\mathbf{0}$ & 1 & . & 0 & $\mathbf{0}$ & $\mathbf{0}$ \\
\hline & $(5,1)$ & 1 & . & $\mathbf{0}$ & $\overline{0}$ & 0 & 1 & • & 0 & 0 & 0 & 1 & . & $\mathbf{0}$ & 0 & $\mathbf{0}$ & 1 & . & 0 & $\mathbf{0}$ & $\mathbf{0}$ \\
\hline & $(1,1)$ & 1 & . & & 0 & 0 & 1 & . & 0 & 0 & 0 & 1 & . & 0 & 0 & 0 & 1 & . & 0 & $\mathbf{0}$ & $\overline{0}$ \\
\hline & $(3,1)$ & 1 & . & & $\mathbf{0}$ & $\mathbf{0}$ & 1 & . & 0 & $\mathbf{0}$ & $\mathbf{0}$ & 1 & . & $\mathbf{0}$ & 0 & $\mathbf{0}$ & 1 & . & 0 & $\mathbf{0}$ & $\mathbf{0}$ \\
\hline 100 & $(3, .5)$ & 1 & . & & $\mathbf{0}$ & 0 & 1 & • & 0 & 0 & 0 & 1 & . & 0 & 0 & $\mathbf{0}$ & 1 & . & 0 & $\mathbf{0}$ & $\mathbf{0}$ \\
\hline & $(4,1)$ & 1 & . & $\mathbf{0}$ & $\mathbf{0}$ & $\mathbf{0}$ & 1 & . & $\mathbf{0}$ & $\mathbf{0}$ & $\mathbf{0}$ & 1 & . & $\mathbf{0}$ & $\mathbf{0}$ & $\mathbf{0}$ & 1 & . & $\mathbf{0}$ & $\mathbf{0}$ & $\mathbf{0}$ \\
\hline & $(4, .5)$ & 1 & . & $\mathbf{0}$ & 0 & 0 & 1 & • & 0 & 0 & 0 & 1 & . & $\mathbf{0}$ & 0 & $\mathbf{0}$ & 1 & . & 0 & 0 & $\mathbf{0}$ \\
\hline & $(5,1)$ & 1 & . & 0 & 0 & $\mathbf{0}$ & 1 & • & 0 & 0 & 0 & 1 & • & 0 & $\mathbf{0}$ & $\mathbf{0}$ & 1 & . & 0 & 0 & $\mathbf{0}$ \\
\hline
\end{tabular}

Table 7(b) Empirical Power of the Tests under Weibull Distribution $(\alpha=0.05)$

\begin{tabular}{|c|c|c|c|c|c|c|c|c|c|c|c|c|c|c|c|c|c|c|c|c|c|}
\hline Sample size(n) & $(\beta, \gamma)$ & \multicolumn{6}{|c|}{$\begin{array}{l}\mathrm{T} \\
W_{n}^{2} S_{n, A}^{1} A_{n}^{2} G_{n}\end{array}$} & & $\mathrm{~S}$ & \multicolumn{2}{|c|}{$\mathrm{t}$} & $\mathrm{a}$ & $\mathrm{t}$ & $\mathrm{i}$ & & s & $\mathrm{t}$ & $\mathrm{i}$ & & & $\mathrm{s}$ \\
\hline \multirow{6}{*}{10} & $(1,1)$ & . & & 5 & 4 & & 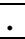 & 9 & 4 & 1 & 7 & . & 9 & 4 & 1 & 4 & . & 5 & 5 & 6 & 5 \\
\hline & $(3,1)$ & • & 9 & 9 & 9 & & • & 9 & 9 & 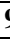 & 8 & . & 9 & 9 & 9 & 9 & . & 5 & 5 & 6 & 5 \\
\hline & $(3, .5)$ & • & 9 & 9 & 9 & & • & 9 & 9 & 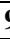 & 7 & . & 9 & 9 & 9 & 2 & . & 9 & 8 & 3 & 0 \\
\hline & $(4,1)$ & 1 & • & $\mathbf{0}$ & $\mathbf{0}$ & & 1 & . & $\mathbf{0}$ & ( & $\mathbf{0}$ & 1 & . & $\mathbf{0}$ & $\mathbf{0}$ & $\mathbf{0}$ & . & 5 & 5 & 6 & 5 \\
\hline & $(4, .5)$ & • & 9 & 9 & 9 & & 1 & . & $\mathbf{0}$ & ( & $\mathbf{0}$ & . & 9 & 9 & 9 & 6 & . & 9 & 8 & 3 & 0 \\
\hline & $(5,1)$ & 1 & • & $\mathbf{0}$ & $\mathbf{0}$ & & 1 & . & $\overline{0}$ & ( & 0 & 1 & - & 0 & $\mathbf{0}$ & $\mathbf{0}$ & . & 5 & 5 & 6 & 5 \\
\hline \multirow{6}{*}{20} & $(1,1)$ & • & 9 & 9 & 7 & & • & 9 & 9 & 7 & 3 & . & 9 & 9 & 4 & 6 & . & 8 & 3 & 7 & 0 \\
\hline & $(3,1)$ & 1 & . & $\mathbf{0}$ & $\mathbf{0}$ & & 1 & 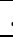 & $\mathbf{0}$ & ( & $\mathbf{0}$ & 1 & . & $\mathbf{0}$ & $\mathbf{0}$ & $\mathbf{0}$ & . & 8 & 3 & 7 & 0 \\
\hline & $(3, .5)$ & 1 & $\dot{ }$ & $\mathbf{0}$ & $\mathbf{0}$ & & 1 & 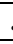 & $\mathbf{0}$ & 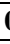 & $\mathbf{0}$ & 1 & . & $\mathbf{0}$ & $\mathbf{0}$ & $\mathbf{0}$ & 1 & . & 0 & 0 & 0 \\
\hline & $(4,1)$ & 1 & • & $\mathbf{0}$ & $\mathbf{0}$ & & 1 & . & $\mathbf{0}$ & 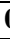 & $\mathbf{0}$ & 1 & - & 0 & $\mathbf{0}$ & $\mathbf{0}$ & . & 8 & 3 & 7 & 0 \\
\hline & $(4, .5)$ & 1 & • & $\mathbf{0}$ & $\mathbf{0}$ & & 1 & . & $\mathbf{0}$ & ( & $\mathbf{0}$ & 1 & . & 0 & $\mathbf{0}$ & $\mathbf{0}$ & 1 & . & 0 & 0 & 0 \\
\hline & $(5,1)$ & 1 & • & 0 & $\mathbf{0}$ & $\mathbf{0}$ & 1 & 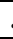 & $\mathbf{0}$ & ( & $\mathbf{0}$ & 1 & . & $\mathbf{0}$ & $\mathbf{0}$ & $\mathbf{0}$ & . & 8 & 3 & 7 & 0 \\
\hline \multirow{8}{*}{25} & $(1,1)$ & • & 9 & 9 & 9 & 8 & $\cdot$ & 9 & 9 & 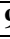 & 7 & . & 9 & 9 & 9 & 1 & . & 9 & 9 & 5 & 3 \\
\hline & $(3,1)$ & 1 & • & $\mathbf{0}$ & $\mathbf{0}$ & & 1 & 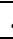 & 0 & ( & $\mathbf{0}$ & 1 & . & 0 & $\mathbf{0}$ & $\mathbf{0}$ & . & 9 & 9 & 5 & 3 \\
\hline & $(3, .5)$ & 1 & $\bullet$ & $\mathbf{0}$ & $\mathbf{0}$ & & 1 & . & 0 & ( & 0 & 1 & $\cdot$ & 0 & $\mathbf{0}$ & 0 & . & 9 & 9 & 9 & 9 \\
\hline & $(4,1)$ & 1 & $\bullet$ & $\mathbf{0}$ & $\mathbf{0}$ & & 1 & 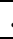 & 0 & ( & $\mathbf{0}$ & 1 & $\dot{\bullet}$ & $\mathbf{0}$ & $\mathbf{0}$ & $\mathbf{0}$ & . & 9 & 9 & 5 & 3 \\
\hline & $(4, .5)$ & 1 & $\dot{-}$ & 0 & $\mathbf{0}$ & & 1 & 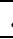 & 0 & I & $\mathbf{0}$ & 1 & $\dot{\bullet}$ & $\mathbf{0}$ & $\mathbf{0}$ & $\mathbf{0}$ & . & 9 & 9 & 9 & 9 \\
\hline & $(5,1)$ & 1 & • & $\mathbf{0}$ & $\mathbf{0}$ & & 1 & 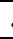 & 0 & ( & $\mathbf{0}$ & 1 & - & 0 & $\mathbf{0}$ & $\mathbf{0}$ & . & 9 & 9 & 5 & 3 \\
\hline & $(1,1)$ & 1 & • & $\underline{\mathbf{0}}$ & $\mathbf{0}$ & $\mathbf{0}$ & 1 & & 0 & ( & $\mathbf{0}$ & . & 9 & 9 & 9 & 8 & . & 9 & 0 & 4 & 6 \\
\hline & $(3,1)$ & 1 & . & $\mathbf{0}$ & $\mathbf{0}$ & $\mathbf{0}$ & 1 & 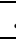 & 0 & 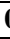 & $\mathbf{0}$ & 1 & . & 0 & $\mathbf{0}$ & $\mathbf{0}$ & . & 9 & 0 & 4 & 6 \\
\hline
\end{tabular}


A Study of Some Tests of Uniformity and Their Performances

\begin{tabular}{|c|c|c|c|c|c|c|c|c|c|c|c|c|c|c|c|c|c|c|c|}
\hline \multirow[t]{4}{*}{30} & $(3, .5)$ & 1 & . & 0 & 0 & $\mathbf{0}$ & 1 & . 0 & $\mathbf{0}$ & 0 & 1 & .0 & $\begin{array}{ll}0 & 0\end{array}$ & $0 \quad 1$ & & 1 & 0 & 0 & 0 \\
\hline & $(4,1)$ & 1 & . & 0 & 0 & 0 & 1 & . 0 & $\mathbf{0}$ & 0 & 1 &.$\quad 0$ & $\begin{array}{ll}0 & 0 \\
\end{array}$ & 0 & 0 & & 90 & 4 & 6 \\
\hline & $(4, .5)$ & 1 & . & $\mathbf{0}$ & $\mathbf{0}$ & $\mathbf{0}$ & 1 & . 0 & 0 & 0 & 1 & & $0 \quad 0$ & 0 & 0 & 1 & .0 & 0 & 0 \\
\hline & $(5,1)$ & 1 & . & 0 & 0 & $\mathbf{0}$ & 1 & . 0 & $\mathbf{0}$ & 0 & 1 & & 0 & 0 & 0 & & 90 & 4 & 6 \\
\hline \multirow{6}{*}{50} & $(1,1)$ & 1 & . & 0 & 0 & $\mathbf{0}$ & 1 & . 0 & 0 & 0 & 1 & & 0 & 0 & 0 & & 94 & 8 & 1 \\
\hline & $(3,1)$ & $\mathbf{1}$ & . & 0 & $\mathbf{0}$ & $\mathbf{0}$ & $\mathbf{1}$ & . 0 & $\mathbf{0}$ & $\mathbf{0}$ & 1 & . 0 & 0 & 0 & 0 & & 94 & 8 & 1 \\
\hline & $(3, .5)$ & 1 & . & 0 & 0 & $\mathbf{0}$ & 1 & . 0 & $\mathbf{0}$ & 0 & 1 & .0 & 0 & 0 & 0 & 1 & .0 & 0 & 0 \\
\hline & $(4,1)$ & 1 & . & $\mathbf{0}$ & 0 & $\mathbf{0}$ & 1 & . 0 & $\mathbf{0}$ & $\mathbf{0}$ & 1 & .0 & 0 & 0 & 0 & & 94 & 8 & 1 \\
\hline & $(4, .5)$ & 1 & . & 0 & 0 & $\mathbf{0}$ & 1 & . 0 & $\mathbf{0}$ & 0 & 1 & . 0 & 0 & 0 & 0 & 1 & 0 & 0 & 0 \\
\hline & $(5,1)$ & 1 & . & 0 & 0 & $\mathbf{0}$ & 1 & . 0 & 0 & 0 & 1 & .80 & 0 & 0 & 0 & & 94 & 8 & 1 \\
\hline \multirow{6}{*}{100} & $(1,1)$ & 1 & . & $\mathbf{0}$ & 0 & $\mathbf{0}$ & 1 & . 0 & $\mathbf{0}$ & 0 & 1 & & 0 & 0 & 0 & 1 & .0 & 0 & 0 \\
\hline & $(3,1)$ & 1 & . & $\mathbf{0}$ & 0 & $\mathbf{0}$ & 1 & . 0 & 0 & 0 & 1 & & 0 & 0 & 0 & 1 & .0 & 0 & 0 \\
\hline & $(3, .5)$ & 1 & . & 0 & 0 & $\mathbf{0}$ & 1 & . 0 & $\mathbf{0}$ & $\mathbf{0}$ & 1 & .0 & 01 & 0 & 0 & 1 & .0 & 0 & 0 \\
\hline & $(4,1)$ & 1 & . & 0 & 0 & 0 & 1 & .0 & $\mathbf{0}$ & $\mathbf{0}$ & 1 & .0 & 0 & 0 & 0 & 1 & .0 & 0 & 0 \\
\hline & $(4, .5)$ & 1 & . & $\mathbf{0}$ & 0 & $\mathbf{0}$ & 1 & .0 & 0 & 0 & 1 & .0 & 0 & 0 & 0 & 1 & .0 & 0 & 0 \\
\hline & $(5,1)$ & 1 & & $\mathbf{0}$ & 0 & $\mathbf{0}$ & 1 & 0 & $\mathbf{0}$ & $\mathbf{0}$ & 1 & .0 & $0 \quad 1$ & 0 & 0 & 1 & .0 & 0 & 0 \\
\hline
\end{tabular}

Fig.1

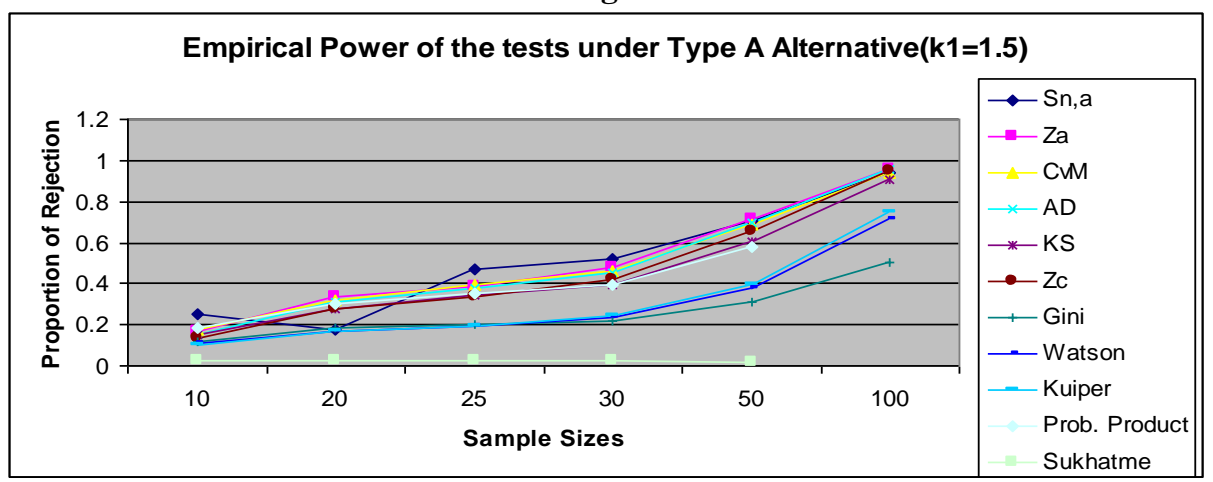

Fig.2

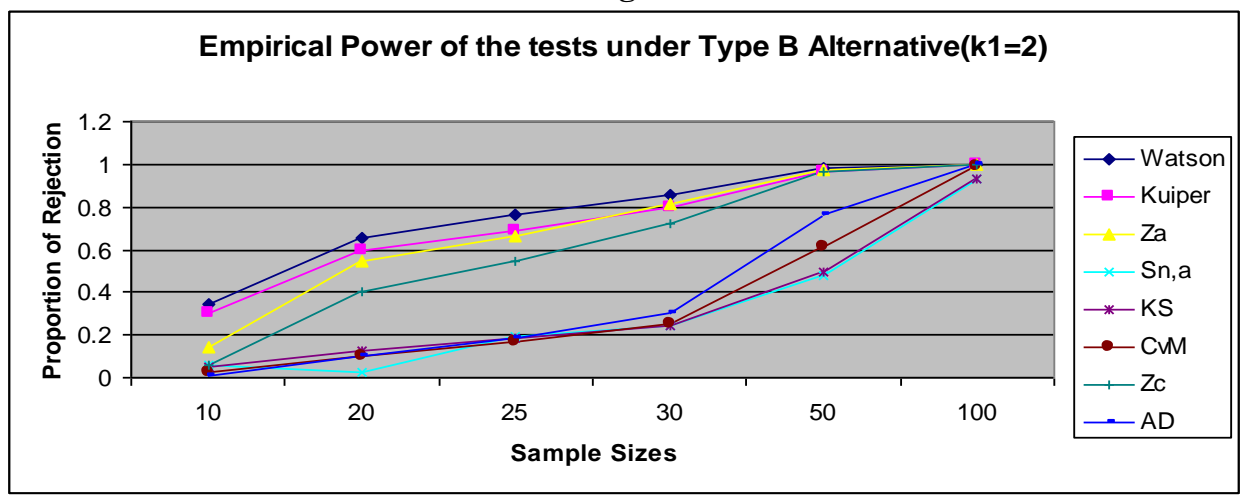

Fig.3

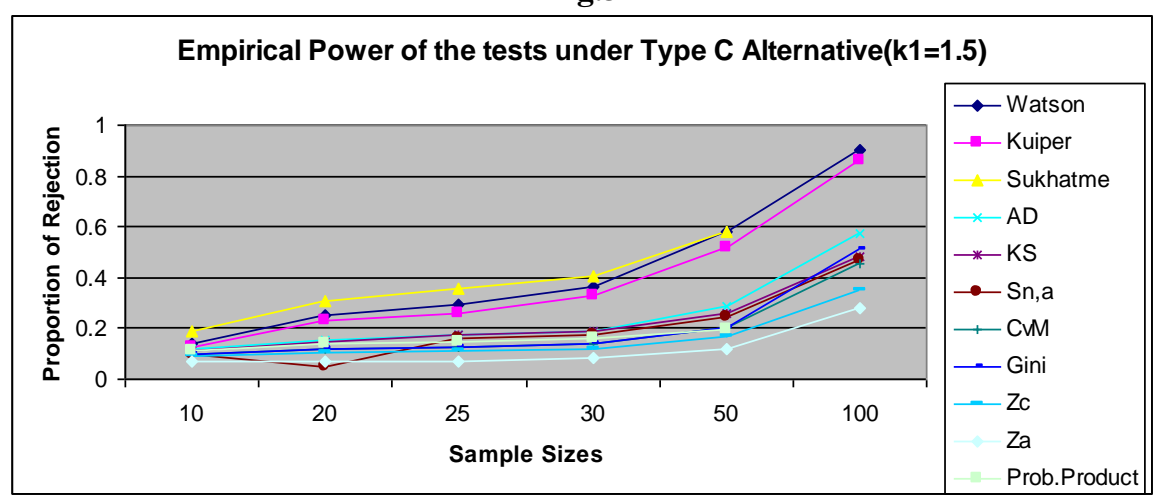

Fig.4 


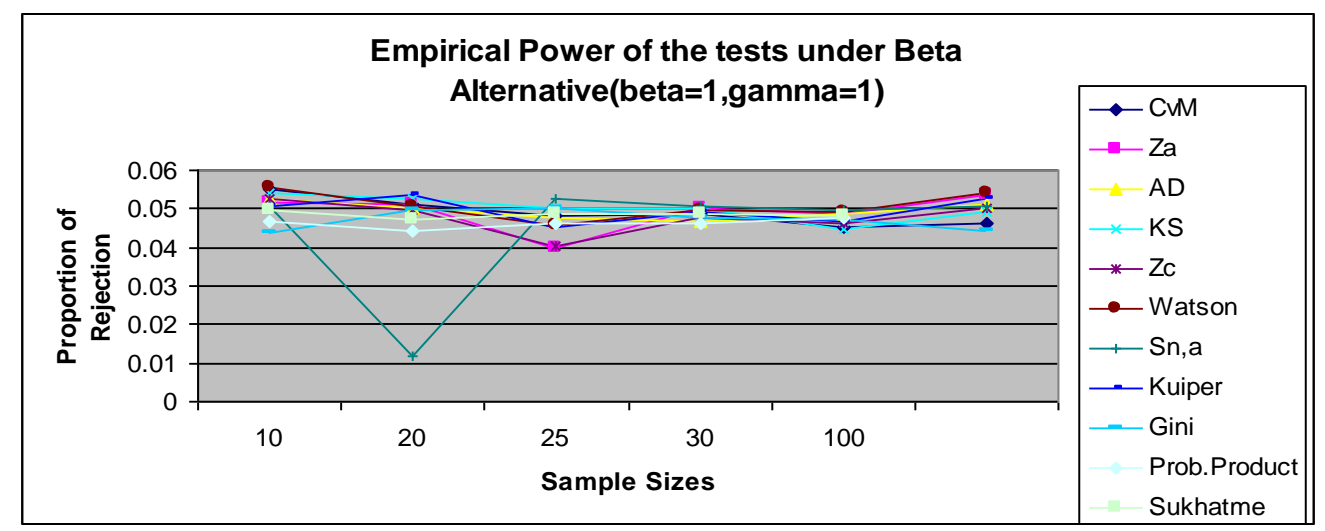

Fig.5

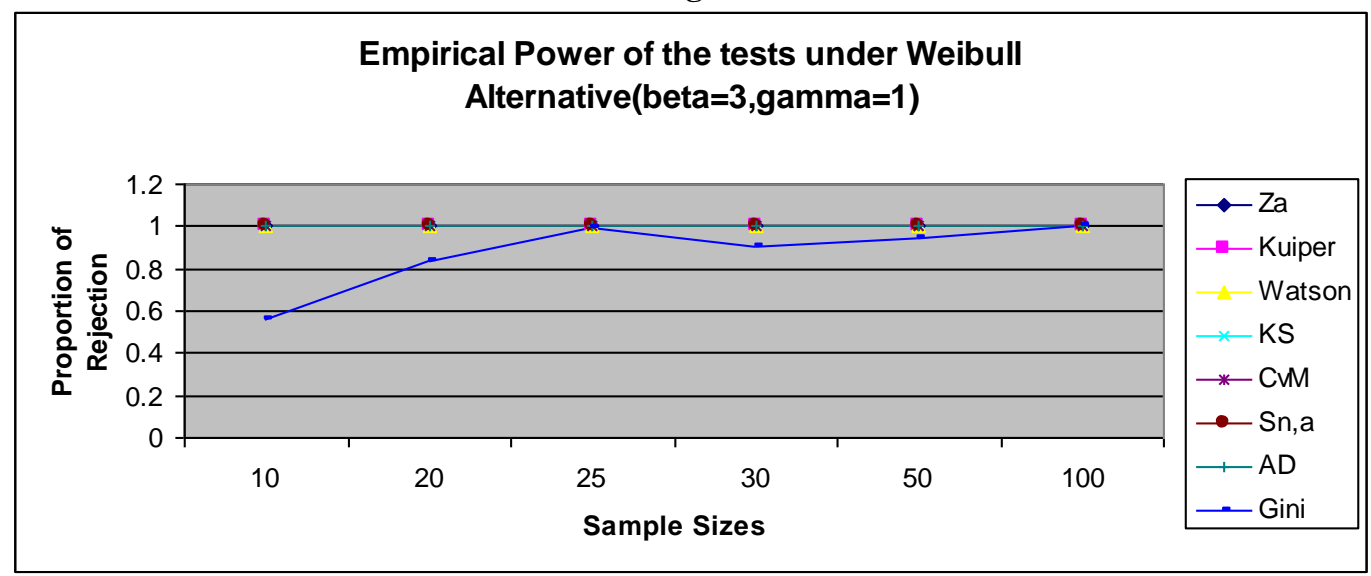

\section{Discussions}

Table 1,2(a) and 2(b) shows the simulated critical values as well as the empirical level of the eleven tests for six different sample sizes. It is seen that all the tests almost satisfy its nominal levels. However, the probability product test is found to be anticonservative in most of the sample sizes. Table 3(a) and 3(b) show the empirical power of eleven tests under the alternative of Type A distribution for six different values of the parameter $\left(\mathrm{k}_{1}\right)$. It is seen that power of all the tests increases as the sample size increases. However, the power of $S_{n, A}^{1} \quad \mathrm{Z}_{\mathrm{A}}, \operatorname{CvM}\left(W_{n}^{2}\right), \operatorname{Gini}\left(G_{n}\right)$ and $\operatorname{AD}\left(A_{n}^{2}\right)$ seems to be higher than the other tests in most of the situation and the power of Sukhatme test is found to be the lowest among all the tests considered here. Also it is found that for some values of the parameter, the CvM and Gini test give the similar power in most of the sample sizes. For large sample sizes as well as for large values of the parameter, the powers of all the tests become exactly one. Table 4(a) and 4(b) show the empirical power of the tests under the alternative of Type B distribution for six different values of the parameter $\left(\mathrm{k}_{1}\right)$.Here also, the power of the tests increases as the sample size increases.However, the powers of Watson $\left(U_{n}^{2}\right), \operatorname{Kuiper}\left(V_{n}\right)$ and $\mathrm{Z}_{\mathrm{A}}$ are found to be higher than the other tests. Also it is seen that as the sample size as well as the value of the parameter increases, the power of $S_{n, A}^{1}, \mathrm{~K}-\mathrm{S}\left(D_{n}\right.$ ), $\operatorname{CvM}\left(W_{n}^{2}\right)$ and $\operatorname{AD}\left(A_{n}^{2}\right)$ tests also increases and it become exactly one.

Table 5(a) and 5(b) show the empirical power of eleven tests under the alternative of Type C distribution for six different values of the parameter $\left(\mathrm{k}_{1}\right)$. Here, it is seen that power of all the tests increases as the sample size increases and also the power becomes exactly one as the sample size increases in most of the tests. The power of Watson $\left(U_{n}^{2}\right), \operatorname{Kuiper}\left(V_{n}\right)$ and Sukhatme $\left(Q_{2}\right)$ tests are found to be higher than the other tests and the power of Probability Product $\left(Q_{1}\right)$ tes is the lowest among all the tests.Also, the power of K-S( $D_{n}$ ), $S_{n, A}^{1}, \operatorname{CvM}\left(W_{n}^{2}\right)$ and $\mathrm{Z}_{\mathrm{C}}$ are found to be in the middle range.

Table 6(a) and 6(b) show the empirical power of eleven tests under the alternative of Beta distribution for six different set of the parameters $(\beta, \gamma)$. It is found that the tests viz., $\operatorname{CvM}\left(W_{n}^{2}\right), \mathrm{Z}_{\mathrm{A}}, \mathrm{AD}\left(A_{n}^{2}\right)$ and $\mathrm{K}-\mathrm{S}\left(D_{n}\right.$ 
)give comparatively higher power than the other tests in all the situations, whereas the Sukhatme $\left(Q_{2}\right)$ and Gini( $\left.G_{n}\right)$ test gives the smallest power in most of the situations. Also the power of $S_{n, A}^{1}$ and Probability Product $\left(Q_{1}\right.$ ) test are found to be in the middle range. The powers of most of the tests become exactly one for large sample sizes and for large values of the parameter.Table 7(a) and 7(b) show the empirical power of the tests under the alternative of Weibull distribution for six different set of the parameters $(\beta, \gamma)$. Here also, it is seen that, the power of all the tests increases as the sample size increases. Out of all the tests considered here, it is seen that, the $\mathrm{Z}_{\mathrm{A}}$ test gives the highest power followed by $\operatorname{Kuiper}\left(V_{n}\right)$, Watson $\left(U_{n}^{2}\right), \mathrm{K}-\mathrm{S}\left(D_{n}\right), \operatorname{CvM}\left(W_{n}^{2}\right), S_{n, A}^{1}$ and $\mathrm{AD}($ $\left.A_{n}^{2}\right)$ and the $\operatorname{Gini}\left(G_{n}\right)$ test gives the smallest power.For large sample sizes as well as for the large values of the parameters, all the tests give the almost same power and finally it becomes exactly one.Also it is found that, for some set of the values of the parameter, the Ginitest gives the same power for a particular sample size.

\section{Conclusion}

Power of $Z_{\mathrm{A}}$ test is found to be higher than the other tests in most of the alternatives except for the alternative of Type C. The tests $\operatorname{CvM}\left(W_{n}^{2}\right), \operatorname{AD}\left(A_{n}^{2}\right)$ and $\mathrm{K}-\mathrm{S}\left(D_{n}\right)$ give almost same power in most of the situations. The power of $S_{n, A}^{1}$, Watson $\left(U_{n}^{2}\right)$ and $\operatorname{Kuiper}\left(V_{n}\right)$ tests are also good for some alternatives. The Sukhatme $\left(Q_{2}\right)$ and Probability Product $\left(Q_{1}\right)$ tests show comparatively less power in most of the situations. Also it is not possible to find out the powers of Sukhatme and Probability Product tests for large sample sizes due to the unavailability of critical values.Finally, we arrive at the conclusion that $\mathrm{Z}_{\mathrm{A}}$ test may be recommended in most of the situations except for the alternative of Type C. We may give second preference to the tests $\mathrm{CvM}$, $\mathrm{AD}, \mathrm{K}-\mathrm{S}$ and $S_{n, A}^{1}$.

\section{References}

[1]. Anderson, T.W. and Darling, D.A.(1954): A test of goodness-of-fit, Jour. Amer. Stat. Assoc., 49, $765-769$.

[2]. Esteban, M.D., Marhuenda, Y., Morales, D. and Sanchez, A. (2006): New Goodness-of-Fit Tests based on Sample Ouantiles, Operations Research Centre, Miguel Hernandez University of Elche, Elche, Spain, 631-642.

[3]. Kolmogorov, A.N. (1933): Sulla Determinazione Empirica Di una Legge di Distribuziane, Giornale Dell’ Instituta Degli Attuari, 4, 83-91.

[4]. Kuiper, N.H. (1960): Tests concerning random points on a circle, Proc. Koninkl. Neder. Akad. Van. Wetenschappen, Series A, 63, 38-47.

[5]. Lemeshko, Yu.B., Blinov, Yu.P. and Lemeshko, S.B. (2016): Goodness of Fit Tests for Uniformity of Probability Distribution Law, Optoelectronics, Instrumentation and Data Processing, 52 (2), 128-140.

[6]. Lewis, P.A.W. (1961): Distribution of the Anderson-Darling statistic, Ann. Math. Stat., 32,1118-1124.

[7]. Noughabi, Alizadeh H., Arghami, N.R. and Borzadaran, Mohtashami G.R. (2014): A test ofGoodness of Fit based on Gini Index, Jour. Turkish Stat. Assoc., 1-10.

[8]. Pearson, K. (1933): On a method of determining whether a sample of size $\mathrm{n}$ supposed havebeen drawn from a parent population having a known probability integral has probabilitybeen drawn at random, Biometrika, 25, 379-410.

[9]. Pearson, E.S. (1938): The probability integral transformation for testing Goodness of Fit andCombining independent test of significance, Biometrika, 30, 134-148.

[10]. Stephens, M.A. (1974): EDF Statistic for Goodness of Fit and Some Comparisons, Jour. Amer. Stat. Assoc., 69, $730-737$.

[11]. Watson, G.S. (1961): Goodness-of-fit on a circle, Biometrika, 48, 109-114.

[12]. Zhang, J. (2002): Powerful goodness-of-fit tests based on the likelihood ratio, JRSR, Series B, 64, $281-294$. 